\title{
Search for light scalar top-quark pair production in final states with two leptons with the ATLAS detector in $\sqrt{s}=7 \mathrm{TeV}$ proton-proton collisions
}

\author{
The ATLAS Collaboration ${ }^{\star}$ \\ CERN, 1211 Geneva 23, Switzerland
}

Received: 21 August 2012 / Revised: 15 October 2012 / Published online: 30 November 2012

(C) CERN for the benefit of the ATLAS collaboration 2012. This article is published with open access at Springerlink.com

\begin{abstract}
A search is presented for the pair production of light scalar top quarks in $\sqrt{s}=7 \mathrm{TeV}$ proton-proton collisions recorded with the ATLAS detector at the Large Hadron Collider. This analysis uses the full data sample collected during 2011 running that corresponds to a total integrated luminosity of $4.7 \mathrm{fb}^{-1}$. Light scalar top quarks are searched for in events with two opposite-sign leptons $(e, \mu)$, large missing transverse momentum and at least one jet in the final state. No excess over Standard Model expectations is found, and the results are interpreted under the assumption that the light scalar top decays to a $b$-quark in addition to an on-shell chargino whose decay occurs through a virtual $W$ boson. If the chargino mass is $106 \mathrm{GeV}$, light scalar top-quark masses up to $130 \mathrm{GeV}$ are excluded for neutralino masses below $70 \mathrm{GeV}$.
\end{abstract}

\section{Introduction}

Weak-scale supersymmetry (SUSY) [1-9] is an extension to the Standard Model (SM) that provides a solution to the instability of the scalar SM sector with respect to new highscale physics. For each known boson or fermion, SUSY introduces a particle with identical quantum numbers except for a difference of half a unit of spin. In the framework of a generic $R$-parity conserving minimal supersymmetric extension of the SM (MSSM) [10-14], SUSY particles are produced in pairs and the lightest supersymmetric particle (LSP) is stable. In a large variety of models, the LSP is the lightest neutralino, $\tilde{\chi}_{1}^{0}$, which is only weakly interacting. The scalar partners of right-handed and left-handed quarks, $\tilde{q}_{R}$ and $\tilde{q}_{L}$, mix to form two mass eigenstates, $\tilde{q}_{1}$ and $\tilde{q}_{2}$, with $\tilde{q}_{1}$ defined to be the lighter one. In the case of the su-

^e-mail: atlas.publications@ @ern.ch persymmetric partner of the top quark ( $\tilde{t}$, stop), large mixing effects can lead to one stop mass eigenstate, $\tilde{t}_{1}$, that is significantly lighter than the other squarks. Depending on the SUSY particle mass spectrum, stop pair production and decay can result in final states topologically similar to $t \bar{t}$ events.

In this Letter, a search for direct stop pair production is presented in $\sqrt{s}=7 \mathrm{TeV}$ proton-proton collisions recorded with the ATLAS detector at the Large Hadron Collider, considering a SUSY particle mass hierarchy such that $m_{t}>$ $m_{\tilde{t}_{1}}>\left(m_{\tilde{\chi}_{1}^{ \pm}}+m_{b}\right)$ and the $\tilde{t}_{1}$ decays exclusively via $b+\tilde{\chi}_{1}^{ \pm}$. The mass of all other supersymmetric particles are set to be above $2 \mathrm{TeV}$, and large stop gauge mixing results in $m_{\tilde{t}_{2}} \gg m_{\tilde{t}_{1}}$ so that only $\tilde{t}_{1}$ pair production is considered. The stop is predominantly right-handed, but this has little effect on the acceptance and efficiency for the final interpretation. The chargino $\left(\tilde{\chi}_{1}^{ \pm}\right.$) mass is set to $106 \mathrm{GeV}$ (above the present exclusion limit of $103.5 \mathrm{GeV}$ [15]) and it is assumed to decay through a virtual $W$ boson $\left(\tilde{\chi}_{1}^{ \pm} \rightarrow W^{*} \tilde{\chi}_{1}^{0}\right)$. The choice of chargino mass is identical to that used in a previous study reported by the CDF experiment [16], thus allowing easy comparison of the CDF and ATLAS results. Stops within a mass range between $110 \mathrm{GeV}$ and $160 \mathrm{GeV}$ would be produced with relatively large cross sections-between $245 \mathrm{pb}$ and $41 \mathrm{pb}$. In this search, dilepton final states $(\ell=e, \mu)$ are considered. Although these events could contribute to an anomaly in the measured $t \bar{t}$ cross section, the relative contribution would be small due to the low transverse momenta of the visible decay products. Events are required to contain at least one energetic jet, large missing transverse momentum $\left(E_{\mathrm{T}}^{\text {miss }}\right)$ and low transverse momenta $\left(p_{\mathrm{T}}\right)$ leptons, to target the light stop final state.

By targeting very light top squarks, this analysis is complementary to other direct stop searches recently presented by the ATLAS experiment $[17,18]$. 


\section{The ATLAS detector}

The ATLAS detector [19] is a multi-purpose particle physics detector with a forward-backward symmetric cylindrical geometry and nearly $4 \pi$ coverage in solid angle. ${ }^{1}$ It contains four superconducting magnet systems, which comprise a solenoid surrounding the inner tracking detector (ID), and the barrel and two end-cap toroids equipping a muon spectrometer. The ID consists of a silicon pixel detector, a silicon microstrip detector (SCT), and a transition radiation tracker (TRT). In the pseudorapidity region $|\eta|<3.2$, high-granularity liquid-argon (LAr) electromagnetic (EM) sampling calorimeters are used. An iron/scintillator tile calorimeter provides coverage for hadron detection over $|\eta|<1.7$. The end-cap and forward regions, spanning $1.5<|\eta|<4.9$, are instrumented with LAr calorimeters for both EM and hadronic measurements. The muon spectrometer surrounds the calorimeters and consists of a system of precision tracking chambers $(|\eta|<2.7)$, and detectors for triggering $(|\eta|<2.4)$.

\section{Simulated event samples}

Monte Carlo (MC) simulated event samples are used to develop and validate the analysis procedure and to evaluate the SM backgrounds in the signal region. Production of top quark pairs is simulated with MC@NLO 4.01 [20-22], using a top-quark mass of $172.5 \mathrm{GeV}$. Samples of $W(\rightarrow \ell v)$ and $Z / \gamma^{*}(\rightarrow \ell \ell$ ), produced with accompanying jets (of both light and heavy flavour), are obtained with ALPGEN 2.14 [23]. Diboson ( $W W, W Z, Z Z)$ production is simulated with HERWIG 6.520 [24] and single top production with MC@NLO 4.01. Fragmentation and hadronisation for the ALPGEN 2.14 and MC@NLO 4.01 samples are performed with HERWIG 6.520, using JIMMY 4.31 [25] for the underlying event. Expected diboson yields are normalised using next-to-leading-order (NLO) QCD predictions obtained with MCFM [26, 27]. The top-quark contribution is normalised to approximate next-to-next-to-leadingorder (NNLO) calculations [28]. The inclusive $W$ and $Z / \gamma^{*}$ production cross sections are normalised to the NNLO cross sections obtained using FEWZ [29]. ALPGEN 2.14 and POWHEG [30] samples are used to assess the systematic uncertainties associated with the choice of generator for $t \bar{t}$ production, and AcerMC [31] samples are used to assess

\footnotetext{
${ }^{1}$ ATLAS uses a right-handed coordinate system with its origin at the nominal interaction point in the centre of the detector and the $z$-axis along the beam pipe. Cylindrical coordinates $(r, \phi)$ are used in the transverse plane, $\phi$ being the azimuthal angle around the beam pipe. The pseudorapidity $\eta$ is defined in terms of the polar angle $\theta$ by $\eta=-\ln \tan (\theta / 2)$
}

the uncertainties associated with initial- and final-state radiation (ISR/FSR) [32]. The choice of the parton distribution functions (PDFs) depends on the generator. CT10 [33] sets are used for all MC@NLO samples. MRST LO** [34] sets are used with HERWIG and PYTHIA, and CTEQ6L1 [35] with ALPGEN 2.14. The stop production models are simulated using PYTHIA 6.425 [36]. Signal cross sections are calculated to next-to-leading order in the strong coupling constant, including the resummation of soft gluon emission at next-to-leading-logarithmic accuracy (NLO + NLL) [37-39]. An envelope of cross-section predictions is defined using the $68 \%$ C.L. ranges of the CTEQ6.6 (including the $\alpha_{S}$ uncertainty) and MSTW 2008 NLO [40] PDF sets, together with independent variations of the factorisation and renormalisation scales by factors of two or one half. The nominal cross-section value is taken to be the midpoint of the envelope and the uncertainty assigned is half the full width of the envelope, following the PDF4LHC recommendations [41]. All MC samples are produced using a GEANT4-based [42] detector simulation [43]. The effect of multiple proton-proton collisions from the same or different bunch crossings is incorporated into the simulation by overlaying additional PYTHIA minimum bias events onto hard-scattering events. Simulated events are weighted to match the distribution of the mean number of interactions per bunch crossing observed in data.

\section{Data and event selection}

The analysis uses the full 2011 proton-proton collision data sample. After applying the beam, detector and data-quality requirements, the data sample corresponds to a total integrated luminosity of $4.7 \mathrm{fb}^{-1}$. Events were triggered using a combination of single and double lepton triggers. The single electron triggers vary with the data-taking period, and the tightest of these has an efficiency of $\sim 97 \%$ for electrons with $p_{\mathrm{T}}>25 \mathrm{GeV}$. The single muon trigger used for all data-taking periods reaches an efficiency plateau of $\sim 75 \%(\sim 90 \%)$ in the barrel (end-caps) for muons with $p_{\mathrm{T}}>20 \mathrm{GeV}$. All efficiencies are quoted with respect to reconstructed leptons, passing the baseline lepton definitions. The double lepton triggers reach similar plateau efficiencies, but at lower $p_{\mathrm{T}}$ thresholds (greater than $17 \mathrm{GeV}$ for electrons passing the dielectron trigger, and greater than $12 \mathrm{GeV}$ for muons passing the dimuon trigger; for the electron-muon trigger the thresholds are 15 and $10 \mathrm{GeV}$ for electrons and muons, respectively). If a lepton has an offline $p_{T}$ above the single lepton trigger plateau threshold in a given event, the relevant single lepton trigger is used. Double lepton triggers are used for events with no such lepton. An exception to this rule is applied in the $\mu \mu$ channel. In this case when one lepton has $p_{\mathrm{T}}>20 \mathrm{GeV}$ and the second 
$p_{\mathrm{T}}>12 \mathrm{GeV}$, a logical OR of both triggers is used to recover efficiency.

Jet candidates are reconstructed using the anti- $k_{t}$ jet clustering algorithm [44] with a radius parameter of 0.4 The input to this algorithm is the three-dimensional energy clusters seeded by calorimeter cells with energy significantly above the noise resulting from the electronics and additional proton-proton interactions (calorimeter clusters). The jet candidate energies are corrected for the effects of calorimeter non-compensation, inhomogeneities and energy loss in material in front of the calorimeter, by using $p_{\mathrm{T}^{-}}$ and $\eta$-dependent calibration factors based on MC simulations and validated with extensive test-beam and collisiondata studies [45]. Furthermore, the reconstructed jet is modified such that the jet direction points to the primary vertex, defined as the vertex with the highest summed track $p_{\mathrm{T}}^{2}$. Only jet candidates with corrected transverse momenta $p_{\mathrm{T}}>20 \mathrm{GeV}$ and $|\eta|<4.5$ are subsequently retained. Jets likely to have arisen from detector noise or cosmic rays are rejected [45]. Electron candidates are required to have $p_{\mathrm{T}}>10 \mathrm{GeV},|\eta|<2.47$, and pass the "medium" shower shape and track selection criteria of Ref. [46]. Muon candidates are reconstructed using either a full muon spectrometer track matched to an ID track, or a muon spectrometer segment matched to an extrapolated ID track [47]. They must be reconstructed with sufficient hits in the pixel, SCT and TRT detectors. They are required to have $p_{\mathrm{T}}>10 \mathrm{GeV}$ and $|\eta|<2.4$.

Following object reconstruction, overlaps between candidate jets and leptons are resolved. Any jet candidate lying within a distance $\Delta R=\sqrt{(\Delta \eta)^{2}+(\Delta \phi)^{2}}=0.2$ of an electron is discarded. Subsequently, any electron or muon candidate remaining within a distance $\Delta R=0.4$ of any surviving jet candidate is discarded.

The measurement of the missing transverse momentum $\mathbf{p}_{\mathrm{T}}^{\text {miss }}$, and its magnitude $E_{\mathrm{T}}^{\text {miss }}$, is based on the transverse momenta of all electrons, muons and jets as described above, and of all calorimeter clusters with $|\eta|<4.5$ not associated to such objects.

Following overlap removal, electrons are further required to have $p_{\mathrm{T}}>17 \mathrm{GeV}$ and to pass the "tight" [46] quality criteria, which places additional requirements on the ratio of calorimetric energy to track momentum, and the fraction of high-threshold hits in the TRT. Electrons are also required to be isolated: the $p_{\mathrm{T}}$ sum of tracks above $1 \mathrm{GeV}$ within a cone of size $\Delta R=0.2$ around each electron candidate (excluding the electron candidates themselves) is required to be less than $10 \%$ of the electron $p_{\mathrm{T}}$. Muons must have $p_{\mathrm{T}}>12 \mathrm{GeV}$ and must be isolated: the $p_{\mathrm{T}}$ sum of tracks within a cone of size $\Delta R=0.2$ around the muon candidate is required to be less than $1.8 \mathrm{GeV}$. Jets are subject to the fur- ther requirements $p_{\mathrm{T}}>25 \mathrm{GeV},|\eta|<2.5$ and a "jet vertex fraction"2 higher than 0.75 .

The top background measurement described below uses a $b$-tagging algorithm [48], which exploits the topological structure of weak $b$ - and $c$-hadron decays inside a candidate jet to identify jets containing a $b$-hadron decay. The nominal $b$-tagging efficiency, computed from $t \bar{t}$ MC events, is on average $60 \%$, with a misidentification (mis-tag) rate for light-quark/gluon jets of less than $1 \%$. To correct small differences in the $b$-tagging efficiency observed in the simulation with respect to the data, a scale factor is applied to all simulated samples.

During part of the data-taking period, a localised electronics failure in the electromagnetic calorimeter created a dead region $(\Delta \eta \times \Delta \phi \approx 1.4 \times 0.2)$. For jets in this region, a correction to their energy is made using the energy depositions in the neighbouring cells, and is propagated to $E_{\mathrm{T}}^{\text {miss }}$. If the energy correction exceeds $10 \mathrm{GeV}$ or $10 \%$ of the $E_{\mathrm{T}}^{\text {miss }}$, the event is discarded. Events with reconstructed electrons in the calorimeter dead region are also rejected.

Events are subject to the following requirements. The primary vertex in the event must have at least five associated tracks and each event must contain exactly two selected leptons (electrons or muons) of opposite sign. Both of these leptons must additionally satisfy the full list of signal lepton requirements, and the dilepton invariant mass, $m_{l l}$, must be greater than $20 \mathrm{GeV}$ across all flavour combinations. In addition, events in the signal region must have at least one jet with $p_{\mathrm{T}}>25 \mathrm{GeV}, E_{\mathrm{T}}^{\text {miss }}>20 \mathrm{GeV}$, missing transverse momentum significance ${ }^{3} E_{\mathrm{T}}^{\text {miss, sig }}>7.5 \mathrm{GeV}^{1 / 2}$ to reject multijet events, and leading lepton $p_{\mathrm{T}}<30 \mathrm{GeV}$ (to provide further rejection of the dominant dileptonic $t \bar{t}$ background). Events in the $e e$ and $\mu \mu$ channels are subject to a further requirement on the dilepton invariant mass to reject events arising from $Z$ production and decay. This selection, summarised in Table 1, has a low signal efficiency, but strong background rejection. The main factor in the efficiency loss is the lowest lepton $p_{\mathrm{T}}$ requirement needed to reach the efficiency plateau of the dilepton triggers. The kinematic acceptance varies between $0.06 \%$ and $0.3 \%$ for a neutralino mass of $55 \mathrm{GeV}$ as the stop mass varies between $112 \mathrm{GeV}$ and $180 \mathrm{GeV}$, and between $0.1 \%$ and $0.003 \%$ for a stop mass of $140 \mathrm{GeV}$ as the neutralino mass varies between $1 \mathrm{GeV}$ and $95 \mathrm{GeV}$ (the detector efficiency for these points, defined as the efficiency for recon-

\footnotetext{
${ }^{2}$ The jet vertex fraction quantifies the fraction of track transverse momentum associated to a jet which comes from the primary vertex. The cut removes jets within the tracker acceptance which originated from uncorrelated soft collisions.

${ }^{3}$ In this paper, $E_{\mathrm{T}}^{\text {miss, sig }}=E_{\mathrm{T}}^{\text {miss }} / \sqrt{H_{\mathrm{T}}}$, where $H_{\mathrm{T}}$ is the scalar sum of the jet and lepton transverse momenta in each event.
} 
Table 1 Signal region, top control region and $Z$ control region requirements in each flavour channel. The $Z$ veto rejects events with $m_{l l}>81 \mathrm{GeV}$ and $m_{l l}<101 \mathrm{GeV}$

\begin{tabular}{|c|c|c|c|}
\hline Requirement & ee channel & $\mu \mu$ channel & $e \mu$ channel \\
\hline \multicolumn{4}{|l|}{ Signal Region } \\
\hline lepton $p_{\mathrm{T}}$ & $>17 \mathrm{GeV}$ & $>12 \mathrm{GeV}$ & $>17(12) \mathrm{GeV}$ for $e(\mu)$ \\
\hline leading lepton $p_{\mathrm{T}}$ & \multicolumn{3}{|c|}{$<30 \mathrm{GeV}$} \\
\hline$m_{l l}$ & $>20$ & veto & $>20 \mathrm{GeV}$ \\
\hline jet $p_{\mathrm{T}}$ & \multicolumn{3}{|c|}{$\geq 1$ jet, $p_{\mathrm{T}}>25 \mathrm{GeV}$} \\
\hline$E_{\mathrm{T}}^{\text {miss }}$ & \multicolumn{3}{|c|}{$>20 \mathrm{GeV}$} \\
\hline$E_{\mathrm{T}}^{\mathrm{miss}, \mathrm{sig}}$ & \multicolumn{3}{|c|}{$>7.5 \mathrm{GeV}^{1 / 2}$} \\
\hline \multicolumn{4}{|l|}{ Top Control Region } \\
\hline lepton $p_{\mathrm{T}}$ & $>17 \mathrm{GeV}$ & $>12 \mathrm{GeV}$ & $>17(12) \mathrm{GeV}$ for $e(\mu)$ \\
\hline leading lepton $p_{\mathrm{T}}$ & \multicolumn{3}{|c|}{$>30 \mathrm{GeV}$} \\
\hline$m_{l l}$ & $>20$ & veto & $>20 \mathrm{GeV}$ \\
\hline jet $p_{\mathrm{T}}$ & \multicolumn{3}{|c|}{$\geq 2(b)$ jets, $p_{\mathrm{T}}>25 \mathrm{GeV}$} \\
\hline$b$-jet $p_{\mathrm{T}}$ & \multicolumn{3}{|c|}{$\geq 1 b$ jet, $p_{\mathrm{T}}>25 \mathrm{GeV}$} \\
\hline$E_{\mathrm{T}}^{\mathrm{miss}}$ & \multicolumn{3}{|c|}{$>20 \mathrm{GeV}$} \\
\hline$E_{\mathrm{T}}^{\text {miss,sig }}$ & \multicolumn{3}{|c|}{$>7.5 \mathrm{GeV}^{1 / 2}$} \\
\hline \multicolumn{4}{|l|}{ Z Control Region } \\
\hline lepton $p_{\mathrm{T}}$ & $>17 \mathrm{GeV}$ & $>12 \mathrm{GeV}$ & $\mathrm{n} / \mathrm{a}$ \\
\hline leading lepton $p_{\mathrm{T}}$ & \multicolumn{2}{|c|}{$<30 \mathrm{GeV}$} & $\mathrm{n} / \mathrm{a}$ \\
\hline$m_{l l}$ & \multicolumn{2}{|c|}{$>81 \mathrm{GeV}$ and $<101 \mathrm{GeV}$} & $\mathrm{n} / \mathrm{a}$ \\
\hline jet $p_{\mathrm{T}}$ & \multicolumn{2}{|c|}{$\geq 1$ jet, $p_{\mathrm{T}}>25 \mathrm{GeV}$} & $\mathrm{n} / \mathrm{a}$ \\
\hline$E_{\mathrm{T}}^{\mathrm{miss}}$ & \multicolumn{2}{|c|}{$>20 \mathrm{GeV}$} & $\mathrm{n} / \mathrm{a}$ \\
\hline$E_{\mathrm{T}}^{\mathrm{miss}, \mathrm{sig}}$ & \multicolumn{2}{|c|}{$>4.0 \mathrm{GeV}^{1 / 2}$} & $\mathrm{n} / \mathrm{a}$ \\
\hline
\end{tabular}

structing events that already enter the kinematic acceptance, is $\sim 40 \%$ ).

\section{Background estimation}

The dominant SM background, after the signal selection requirements, arises from $t \bar{t}$ events where both top quarks decay leptonically, with the next most significant background being $Z / \gamma^{*}+$ jets. Single top, $W+$ jets, diboson and multijet events give much smaller expected contributions.

The fully leptonic $t \bar{t}$ background in the signal region is obtained by extrapolating the number of $t \bar{t}$ events measured in a suitable control region (CR), after correcting for contamination from non- $t \bar{t}$ events, into the signal region (SR). This extrapolation, detailed in Eq. (1), uses the ratio of the number of simulated $t \bar{t}$ events in the signal region to those in the control region:

$\left(N_{t \bar{t}}\right)_{\mathrm{SR}}=\left[\left(N_{\text {data }}\right)_{\mathrm{CR}}-\left(N_{\text {non }-t \bar{t}, \mathrm{MC}}\right)_{\mathrm{CR}}\right] \frac{\left(N_{t \bar{t}, \mathrm{MC}}\right)_{\mathrm{SR}}}{\left(N_{t \bar{t}, \mathrm{MC}}\right)_{\mathrm{CR}}}$

The CR is designed to give an event sample dominated by top events, whilst minimising signal contamination. It is further chosen to be kinematically similar to the signal region to minimise systematic uncertainties due to extrapolation. Selection requirements for the top control region are summarised in Table 1. In this analysis, models with small stop-chargino mass difference are considered, and hence soft $b$-jets are expected in the signal events which are not efficiently tagged. By requiring a $b$-jet in the top control region a high-purity sample of top events is obtained. The signal contamination in the considered models is typically of the order of a few per cent, rising to $30 \%$ for models with $m_{\tilde{\chi}_{1}^{0}}=1 \mathrm{GeV}$ and high $m_{\tilde{t}_{1}}$. The percentage of SM, non- $t \bar{t}$ events in the CR is less than $5 \%$ across all channels. The resulting $t \bar{t}$ background contributions are consistent with the expected MC yields in all channels within the uncertainties. Signal contamination is taken into account when setting the exclusion limit in the next section by including, for each signal model, the expected signal yield in the top control region in the $\left(N_{\text {non- } t \bar{t}, \mathrm{MC}}\right)_{\mathrm{CR}}$ term in Eq. (1).

The contribution from $Z / \gamma^{*}+$ jets events to the signal region (from $e e$ and $\mu \mu$ events) is evaluated in a similar way. Data are used to obtain the normalisation of the $Z / \gamma^{*}$ background in a suitable $\mathrm{CR}$ and $\mathrm{MC}$ is used to extrapolate from CR to SR using an equation analogous to Eq. (1). This method is used separately for each of the $e e$ and $\mu \mu$ channels (with selection requirements for the $Z \mathrm{CR}$ as summarised in Table 1), whereas the contribution to $e \mu$ (including those from $\left.Z / \gamma^{*} \rightarrow \tau \tau\right)$ is taken directly from the MC simulation due to the limited number of events in the CR. 
The contamination from non- $Z / \gamma^{*}+$ jets SM events in the $\mathrm{CR}$ is less than $5 \%$, and the signal contamination less than $4 \%$. The resulting $Z$ background contributions are consistent with the expected MC yields in the $e e$ and $\mu \mu$ channels within the uncertainties. The effect of signal contamination of the $Z$ control region on the final exclusion limit can be neglected to a very good approximation.

Single top, $W+$ jets (including heavy-flavour contributions) and diboson backgrounds are evaluated in the signal region directly from the MC simulation. The estimated contribution from $W+$ jets has been cross-checked using a data-driven technique (an extension of the "template fit", described below), and found to be in good agreement.

The tight requirement on $E_{\mathrm{T}}^{\text {miss,sig }}$ heavily suppresses the multijet background. A data-driven template fit technique is used to verify that this background is small, and to assign an uncertainty on the yield in the signal region. The isolation requirements on the electrons and muons are reversed to enhance the multijet content of selected events. The requirements are inverted in the signal region, prior to application of the $E_{\mathrm{T}}^{\text {miss,sig }}$ requirement. The shape of the $E_{\mathrm{T}}^{\text {miss,sig }}$ distribution in data for this inverted selection (after subtracting the dominantly electroweak background using the MC simulation) is then compared to the equivalent distribution in data for the "normal" isolation requirements in order to validate that inverting the lepton isolation does not distort the shape of the distribution. The "normal" and "inverted" shapes were found to agree very closely for the full range of distributions considered in the analysis. The inverted $E_{\mathrm{T}}^{\text {miss,sig }}$ distribution is then renormalised to match the distribution after nominal isolation requirements. Passing this correctly normalised template through the remaining requirements gives the multijet yield in the signal region. It is found to be small in all channels, making up less than $2 \%$ of the total background.

\section{Systematic uncertainties}

The total systematic uncertainty on the expected background in the combined flavour channel (the sum of $e e, e \mu$ and $\mu \mu$ events) is $9.8 \%$, and is dominated by the uncertainties on the two largest backgrounds (dileptonic $t \bar{t}$ and $Z+$ jet events). The largest source of systematic uncertainty on the $t \bar{t}$ background evaluation is the uncertainty on the jet energy scale (JES), with smaller contributions coming from the jet energy resolution (JER) uncertainty [45], the theory and MC modelling uncertainties (using the prescriptions described in Ref. [49]), the systematic uncertainties on the $b$-tagging efficiency [48], and the uncertainty arising from the limited numbers of MC and data events. Uncertainties [46, 50, 51] in lepton reconstruction and identification (momentum and energy scales, resolutions and efficiencies) give smaller contributions.

The primary source of uncertainty on the $Z / \gamma^{*}+$ jets background estimate in the combined flavour channel is the jet energy resolution uncertainty, with smaller contributions coming from the statistical and jet energy scale uncertainties. Theoretical uncertainties on the $Z / \gamma^{*}+$ jets background are investigated by varying the PDF and renormalisation scales. An uncertainty on the luminosity of $3.9 \%$ $[52,53]$ is included in the systematic uncertainty calculation for backgrounds taken directly from the MC simulation. The dominant uncertainties on these backgrounds are the jet energy scale and statistical uncertainties. The systematic uncertainty on the multijet yield is obtained by varying the range in which the template fit is performed, and using the maximum deviation of the final yield to assign the uncertainty.

In the considered $m_{\tilde{\chi}_{1}^{0}-m_{\tilde{t}_{1}}}$ mass plane the theoretical uncertainty on each of the signal cross sections is approximately $16 \%$. These arise from considering the crosssection envelope defined using the $68 \%$ C.L. ranges of the CTEQ6.6 and MSTW 2008 NLO PDF sets, and independent variations of the factorisation and renormalisation scales (see Sect. 3). Further uncertainties on the numbers of predicted signal events arise from the JES uncertainty (7-15\%), the JER uncertainty (1-7\%), the luminosity uncertainty (3.9\%), the uncertainties on calorimeter energy clusters used to calculate $E_{\mathrm{T}}^{\text {miss }}(2-6 \%)$, the statistical uncertainty from finite MC event samples (4-20\%) and smaller contributions from uncertainties on lepton reconstruction and identification, where the quoted ranges display the maximum variation observed using all signal models considered in this analysis.

\section{Results and interpretation}

Table 2 shows the data observations in the signal regions in each flavour channel, and in the combined flavour channel, along with the evaluated background contributions. Good agreement is observed across all channels, and the absence of evidence for light scalar top production allows a limit to be set on the visible cross section for non-SM physics, $\sigma_{\text {vis }}=\sigma \times \epsilon \times A$, for which this analysis has an efficiency $\epsilon$ and acceptance $A$. The limits are calculated using the modified frequentist $C L_{s}$ prescription [54] by comparing the number of observed events in data with the SM and SMplus-signal expectations.

All systematic uncertainties and their correlations are taken into account via nuisance parameters using a profile likelihood technique [55]. In Fig. 1, the leading lepton $p_{\mathrm{T}}$ distributions in the $e e$ and $\mu \mu$ channels are illustrated along 
Table 2 The expected and observed numbers of events in the signal region for each flavour channel. In the combined flavour column ("all"), the statistical uncertainty (first uncertainty quoted, includes the $\mathrm{MC}$ and data statistical errors) on the various background estimates have each been added in quadrature whilst the systematic uncertainties (second uncertainty quoted) have been combined taking into account the correlations between background sources. Observed and expected upper limits at $95 \%$ confidence level on the visible cross section $\sigma_{\text {vis }}=\sigma \times A \times \epsilon$ are also shown.

The expected signal yields and statistical uncertainties on the yields are quoted for the two mass points illustrated in the figures

\begin{tabular}{lllll}
\hline & $e e$ & $e \mu$ & $\mu \mu$ & all \\
\hline$t \bar{t}$ & $44 \pm 4 \pm 5$ & $139 \pm 7 \pm 22$ & $111 \pm 8 \pm 10$ & $293 \pm 12 \pm 34$ \\
$Z / \gamma^{*}+$ jets & $5 \pm 1 \pm 2$ & $23 \pm 2 \pm 8$ & $48 \pm 16 \pm 27$ & $76 \pm 16 \pm 27$ \\
Single top & $3 \pm 0.5 \pm 1$ & $12 \pm 1 \pm 2$ & $12 \pm 1 \pm 2$ & $28 \pm 2 \pm 5$ \\
$W+$ jets & $3 \pm 3 \pm 3$ & $5 \pm 2 \pm 1$ & $6 \pm 2 \pm 1$ & $13 \pm 3 \pm 3$ \\
Diboson & $4 \pm 0.4 \pm 0.5$ & $9 \pm 0.7 \pm 2$ & $10 \pm 0.7 \pm 1$ & $22 \pm 1 \pm 3$ \\
Multijet & $2.9_{-2.9}^{+3.2 \pm 2.2}$ & $2.0 \pm 1.4 \pm 0.3$ & $3.0 \pm 2.8 \pm 0.3$ & $8.0 \pm 3.7 \pm 2.3$ \\
Total & $61 \pm 6 \pm 6$ & $189 \pm 8 \pm 21$ & $190 \pm 19 \pm 31$ & $440 \pm 21 \pm 43$ \\
Data & 48 & 188 & 195 & 431 \\
$\sigma_{\text {vis }}(\exp$. limit $)[\mathrm{fb}]$ & 4.9 & 11.1 & 16.2 & 22.0 \\
$\sigma_{\text {vis }}($ obs. limit $)[\mathrm{fb}]$ & 3.3 & 10.9 & 16.9 & 21.0 \\
$\left(m_{\tilde{t}_{1}}, m_{\tilde{\chi}_{1}^{0}}\right)=(112,55) \mathrm{GeV}$ & $44.1 \pm 4.8$ & $137 \pm 8$ & $322 \pm 13$ \\
$\left(m_{\tilde{t}_{1}}, m_{\tilde{\chi}_{1}^{0}}\right)=(160,55) \mathrm{GeV}$ & $8.8 \pm 1.5$ & $31.4 \pm 2.7$ & $36.5 \pm 2.9$ & $76.6 \pm 4.3$ \\
\hline
\end{tabular}

with the $E_{\mathrm{T}}^{\text {miss }}$ and $E_{\mathrm{T}}^{\text {miss,sig }}$ distributions of the data and simulated events in the signal region (with the background normalisations set to their nominal values).

The observed data yield is in good agreement with the SM prediction in the combined flavour channel given in Table 2 .

The results in the combined channel are used to place exclusions at $95 \%$ confidence level in the $m_{\tilde{t}_{1}}-m_{\tilde{\chi}_{1}^{0}}$ mass plane, using the $\mathrm{CL}_{\mathrm{s}}$ method. The resulting $95 \%$ confidence level expected (dashed) and observed (solid) limits are shown in Fig. 2. Neutralino masses down to $1 \mathrm{GeV}$ are considered, since there is no LEP limit on the neutralino mass in the MSSM for the case that the lightest neutralino is predominantly bino in nature. A bino-dominated lightest neutralino is favoured by the recent LHC Higgs search results.

The observed limits represent a significant extension of the CDF limit [16] for a chargino mass of $106 \mathrm{GeV}$ to smaller chargino minus neutralino mass difference (the ATLAS limit extends up to a neutralino mass of $70 \mathrm{GeV}$ for a stop mass of $130 \mathrm{GeV}$, whilst the CDF limit extends up to a neutralino mass of $46 \mathrm{GeV}$ ).

The limit on the stop mass for neutralino masses of $45 \mathrm{GeV}(135 \mathrm{GeV})$ is comparable to the equivalent CDF limit. Increasing the chargino mass by $15 \mathrm{GeV}$ leads to a modest shift of the exclusion limit to higher values of the neutralino mass, with the reach in stop mass being enhanced to a lesser degree due to the falling stop production cross section. For example, for a model with $m_{\tilde{t}_{1}}=130 \mathrm{GeV}$, $m_{\tilde{\chi}_{1}^{ \pm}}=120 \mathrm{GeV}$ and $m_{\tilde{\chi}_{1}^{0}}=60 \mathrm{GeV}$, the value of $A \times \epsilon$
$(0.032 \%)$ is slightly higher than for the equivalent model with $m_{\tilde{\chi}_{1}^{ \pm}}=106 \mathrm{GeV}(0.026 \%)$.

\section{Conclusions}

A search for light top squarks has been performed in the dilepton final state. SM backgrounds have been evaluated using a combination of data-driven techniques and MC simulation. Good agreement is observed between data and the SM prediction in all three flavour channels. The results are interpreted in the $m_{\tilde{t}_{1}}-m_{\tilde{\chi}_{1}^{0}}$ plane with the chargino mass set to $106 \mathrm{GeV}$, and with the assumption that the decay $\tilde{t}_{1} \rightarrow b \tilde{\chi}_{1}^{ \pm}$occurs $100 \%$ of the time, followed by decay via a virtual $W\left(\tilde{\chi}_{1}^{ \pm} \rightarrow W^{*} \tilde{\chi}_{1}^{0}\right)$ with an $11 \%$ branching ratio (per flavour channel) to decay leptonically. A lower limit at $95 \%$ confidence level is set on the stop mass in this plane using the combined flavour channel. This excludes stop masses up to $130 \mathrm{GeV}$ (for neutralino masses between $1 \mathrm{GeV}$ and $70 \mathrm{GeV}$ ). This limit exceeds that set by the CDF Collaboration for the same scenario [16].

Acknowledgements We thank CERN for the very successful operation of the LHC, as well as the support staff from our institutions without whom ATLAS could not be operated efficiently.

We acknowledge the support of ANPCyT, Argentina; YerPhI, Armenia; ARC, Australia; BMWF, Austria; ANAS, Azerbaijan; SSTC, Belarus; CNPq and FAPESP, Brazil; NSERC, NRC and CFI, Canada; CERN; CONICYT, Chile; CAS, MOST and NSFC, China; COLCIENCIAS, Colombia; MSMT CR, MPO CR and VSC CR, Czech Republic; DNRF, DNSRC and Lundbeck Foundation, Denmark; EPLANET 


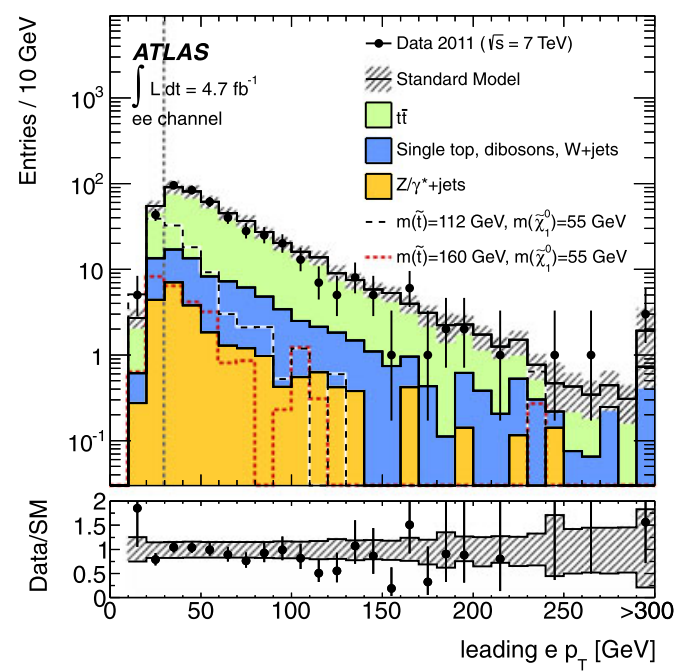

(a)

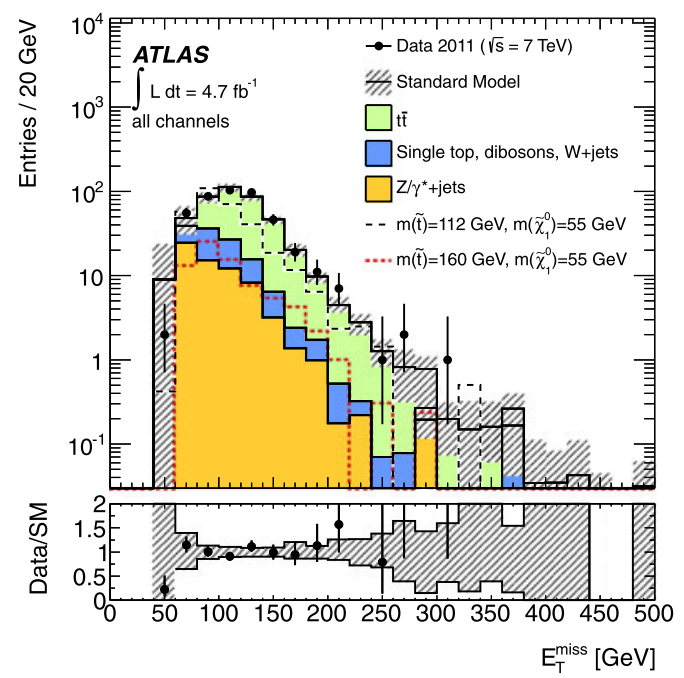

(c)

Fig. 1 The leading electron and muon $p_{\mathrm{T}}$ distributions in the same flavour (a) $e e$ and (b) $\mu \mu$ channels, before the requirement on the leading lepton $p_{\mathrm{T}}$ (which is marked by the dashed vertical line), and (c) the $E_{\mathrm{T}}^{\text {miss }}$ distribution and (d) $E_{\mathrm{T}}^{\text {miss,sig }}$ distribution after all signal region requirements. The data and evaluated background components are shown. The hashed band indicates the total experimental uncertainty on the expectation. The dashed lines give the expectations for

and ERC, European Union; IN2P3-CNRS, CEA-DSM/IRFU, France; GNSF, Georgia; BMBF, DFG, HGF, MPG and AvH Foundation, Germany; GSRT, Greece; ISF, MINERVA, GIF, DIP and Benoziyo Center, Israel; INFN, Italy; MEXT and JSPS, Japan; CNRST, Morocco; FOM and NWO, Netherlands; RCN, Norway; MNiSW, Poland; GRICES and FCT, Portugal; MERYS (MECTS), Romania; MES of Russia and ROSATOM, Russian Federation; JINR; MSTD, Serbia; MSSR, Slovakia; ARRS and MVZT, Slovenia; DST/NRF, South Africa; MICINN, Spain; SRC and Wallenberg Foundation, Sweden; SER, SNSF and

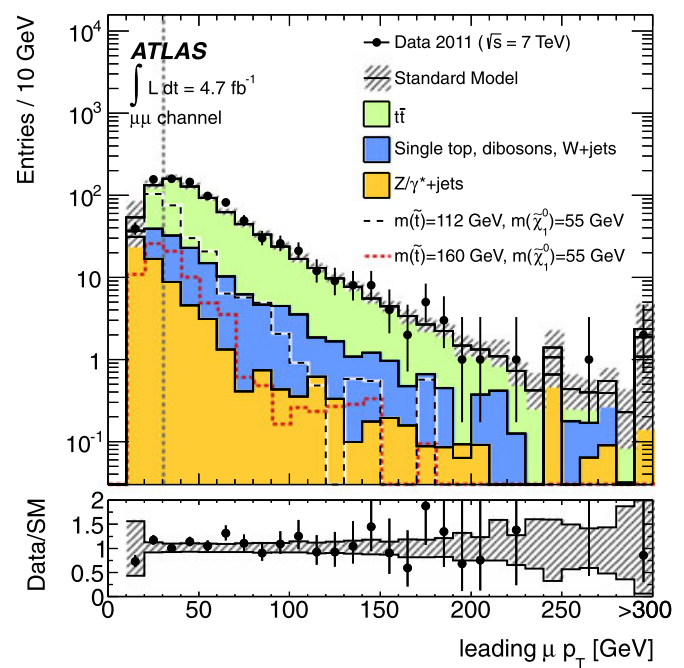

(b)

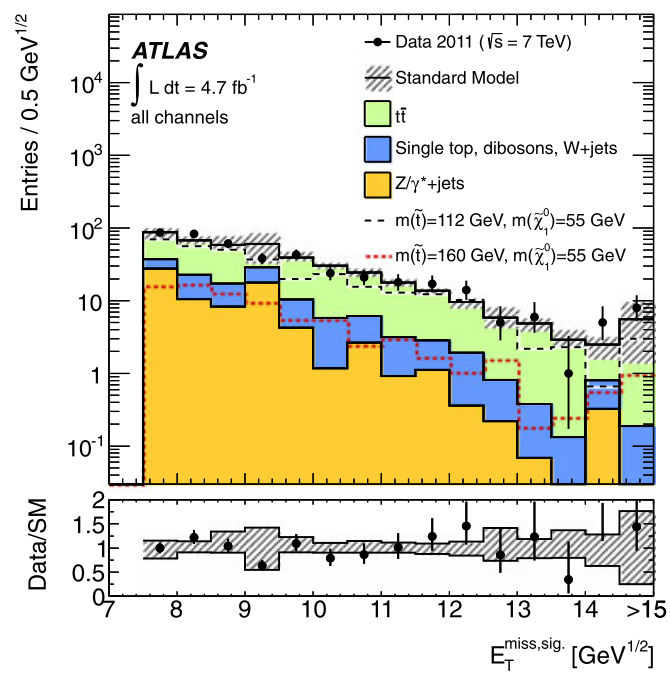

(d)

signal models with stop masses of $112 \mathrm{GeV}$ and $160 \mathrm{GeV}$, and a neutralino mass of $55 \mathrm{GeV}$. The last histogram bins in (a) and (b) include the integrals of all events with $p_{\mathrm{T}}>300 \mathrm{GeV}$. The final bin in (d) includes all events with an $E_{\mathrm{T}}^{\mathrm{miss} \text {, sig }}$ of at least $15 \mathrm{GeV}^{1 / 2}$. The bottom panels show the ratio of the data to the expected background (points) and the systematic uncertainty on the background (hashed area)

Cantons of Bern and Geneva, Switzerland; NSC, Taiwan; TAEK, Turkey; STFC, the Royal Society and Leverhulme Trust, United Kingdom; DOE and NSF, United States of America.

The crucial computing support from all WLCG partners is acknowledged gratefully, in particular from CERN and the ATLAS Tier-1 facilities at TRIUMF (Canada), NDGF (Denmark, Norway, Sweden), CC-IN2P3 (France), KIT/GridKA (Germany), INFN-CNAF (Italy), NL-T1 (Netherlands), PIC (Spain), ASGC (Taiwan), RAL (UK) and BNL (USA) and in the Tier-2 facilities worldwide. 


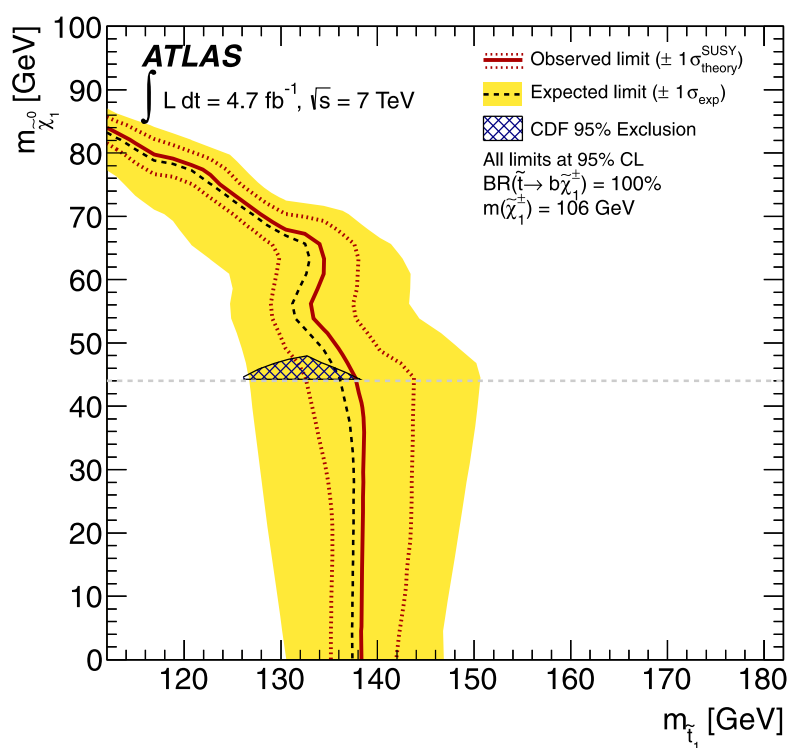

Fig. $295 \%$ exclusion limit in the $m_{\tilde{t}_{1}}-m_{\tilde{\chi}_{1}^{0}}$ mass plane, with $m_{\tilde{\chi}_{1}^{ \pm}}=106 \mathrm{GeV}$. The dashed and solid lines show the $95 \%$ C.L. expected and observed limits, respectively, including all uncertainties except for the theoretical signal cross-section uncertainty (PDF and scale). The band around the expected limit shows the $\pm 1 \sigma$ result. The dotted $\pm 1 \sigma$ lines around the observed limit represent the results obtained when moving the nominal signal cross section up or down by the theoretical uncertainty. Illustrated also is the region excluded at the $95 \%$ C.L. by CDF [16], where the lowest neutralino mass considered was $44 \mathrm{GeV}$, indicated by the horizontal dotted line

Open Access This article is distributed under the terms of the Creative Commons Attribution License which permits any use, distribution, and reproduction in any medium, provided the original author(s) and the source are credited.

\section{References}

1. H. Miyazawa, Prog. Theor. Phys. 36(6), 1266-1276 (1966)

2. P. Ramond, Phys. Rev. D 3, 2415-2418 (1971)

3. Y.A. Golfand, E.P. Likhtman, JETP Lett. 13, 323-326 (1971) [Pisma Zh. Eksp. Teor. Fiz. 13, 452-455 (1971)]

4. A. Neveu, J.H. Schwarz, Nucl. Phys. B 31, 86-112 (1971)

5. A. Neveu, J.H. Schwarz, Phys. Rev. D 4, 1109-1111 (1971)

6. J. Gervais, B. Sakita, Nucl. Phys. B 34, 632-639 (1971)

7. D.V. Volkov, V.P. Akulov, Phys. Lett. B 46, 109-110 (1973)

8. J. Wess, B. Zumino, Phys. Lett. B 49, 52 (1974)

9. J. Wess, B. Zumino, Nucl. Phys. B 70, 39-50 (1974)

10. P. Fayet, Phys. Lett. B 64, 159 (1976)

11. P. Fayet, Phys. Lett. B 69, 489 (1977)

12. G.R. Farrar, P. Fayet, Phys. Lett. B 76, 575-579 (1978)

13. P. Fayet, Phys. Lett. B 84, 416 (1979)

14. S. Dimopoulos, H. Georgi, Nucl. Phys. B 193, 150 (1981)

15. L.E.P. SUSY Working Group (ALEPH, DELPHI, L3, OPAL), Note LEPSUSYWG/01-03.1, http://lepsusy.web.cern.ch/lepsusy/ Welcome.html

16. CDF Collaboration, Phys. Rev. Lett. 104, 251801 (2010)
17. ATLAS Collaboration, arXiv:1208.1447 (2012)

18. ATLAS Collaboration, arXiv: 1208.2590 (2012)

19. ATLAS Collaboration, J. Instrum. 3, S08003 (2008)

20. S. Frixione, B.R. Webber, J. High Energy Phys. 06, 029 (2002)

21. S. Frixione, P. Nason, B.R. Webber, J. High Energy Phys. 08, 007 (2003)

22. S. Frixione, E. Laenen, P. Motylinski, J. High Energy Phys. 03, 092 (2006)

23. M.L. Mangano, M. Moretti, F. Piccinini, R. Pittau, A.D. Polosa, J. High Energy Phys. 07, 001 (2003)

24. G. Corcella et al., J. High Energy Phys. 01, 010 (2001)

25. J. Butterworth, J. Forshaw, M. Seymour, Z. Phys. C 72, 637-646 (1996)

26. J.M. Campbell, R.K. Ellis, Phys. Rev. D 60, 113006 (1999)

27. J.M. Campbell, R.K. Ellis, C. Williams, J. High Energy Phys. 07, $018(2011)$

28. M. Aliev, H. Lacker, U. Langenfeld, S. Moch, P. Uwer et al., Comput. Phys. Commun. 182, 1034-1046 (2011)

29. R. Gavin, Y. Li, F. Petriello, S. Quackenbush, Comput. Phys. Commun. 182, 2388-2403 (2011)

30. S. Frixione, P. Nason, C. Oleari, J. High Energy Phys. 11, 070 (2007)

31. B.P. Kersevan, E. Richter-Was, arXiv:hep-ph/0405247 v1 (2004)

32. ATLAS Collaboration, arXiv:1203.5015 (2012)

33. H.-L. Lai, M. Guzzi, J. Huston, Z. Li, P.M. Nadolsky et al., Phys. Rev. D 82, 074024 (2010)

34. A. Sherstnev, R. Thorne, arXiv:0807.2132 (2008)

35. J. Pumplin et al., J. High Energy Phys. 07, 012 (2002)

36. T. Sjostrand, S. Mrenna, P. Skands, J. High Energy Phys. 05, 026 (2006)

37. W. Beenakker, M. Kramer, T. Plehn, M. Spira, P.M. Zerwas, Nucl. Phys. B 515, 3-14 (1998)

38. W. Beenakker, S. Brensing, M. Kramer, A. Kulesza, E. Laenen, I. Niessen, J. High Energy Phys. 1008, 098 (2010)

39. W. Beenakker, S. Brensing, M. Kramer, A. Kulesza, E. Laenen et al., Int. J. Mod. Phys. A26, 2637-2664 (2011)

40. A.D. Martin, W.J. Stirling, R.S. Thorne, G. Watt, Eur. Phys. J. C 63, 189-285 (2009)

41. M. Botje, J. Butterworth, A. Cooper-Sarkar, A. de Roeck, J. Feltesse et al., arXiv:1101.0538 (2011)

42. S. Agostinelli et al., Nucl. Instrum. Methods A 506, 250 (2003)

43. ATLAS Collaboration, Eur. Phys. J. C 70, 823 (2010)

44. M. Cacciari, G.P. Salam, G. Soyez, J. High Energy Phys. 04, 063 (2008)

45. ATLAS Collaboration, arXiv: 1112.6426 (2011)

46. ATLAS Collaboration, Eur. Phys. J. C 72, 2012 (1909)

47. ATLAS Collaboration, ATLAS-CONF-2011-063. https://cdsweb. cern.ch/record/1345743 (2011)

48. ATLAS Collaboration, ATLAS-CONF-2011-102. https://cdsweb. cern.ch/record/1369219 (2011)

49. ATLAS Collaboration, Phys. Lett. B 707, 459 (2012)

50. ATLAS Collaboration, ATLAS-CONF-2011-063. https://cdsweb. cern.ch/record/1345743 (2011)

51. ATLAS Collaboration, ATLAS-CONF-2011-021. https://cdsweb. cern.ch/record/1336750 (2011)

52. ATLAS Collaboration, Eur. Phys. J. C 71, 1630 (2011). arXiv: 1101.2185

53. ATLAS Collaboration, ATLAS-CONF-2011-116. http://cdsweb. cern.ch/record/1376384 (2011)

54. A.L. Read, J. Phys. G 28, 2693-2704 (2002)

55. G. Cowan, K. Cranmer, E. Gross, O. Vitells, Eur. Phys. J. C 71, $1554(2011)$ 


\section{The ATLAS Collaboration}

G. Aad $^{47}$, T. Abajyan ${ }^{20}$, B. Abbott ${ }^{110}$, J. Abdallah ${ }^{11}$, S. Abdel Khalek ${ }^{114}$, A.A. Abdelalim ${ }^{48}$, O. Abdinov ${ }^{10}$, R. Aben ${ }^{104}$, B. $\mathrm{Abi}^{111}$, M. Abolins ${ }^{87}$, O.S. AbouZeid ${ }^{157}$, H. Abramowicz ${ }^{152}$, H. Abreu ${ }^{135}$, E. Acerbi ${ }^{88 a, 88 b}$, B.S. Acharya ${ }^{163 a, 163 b}$, L. Adamczyk ${ }^{37}$, D.L. Adams ${ }^{24}$, T.N. Addy ${ }^{55}$, J. Adelman ${ }^{175}$, S. Adomeit ${ }^{97}$, P. Adragna ${ }^{74}$, T. Adye ${ }^{128}$, S. Aefsky ${ }^{22}$, J.A. Aguilar-Saavedra ${ }^{123 b, a}$, M. Agustoni ${ }^{16}$, M. Aharrouche ${ }^{80}$, S.P. Ahlen ${ }^{21}$, F. Ahles ${ }^{47}$, A. Ahmad ${ }^{147}$, M. Ahsan ${ }^{40}$, G. Aielli1 ${ }^{132 a, 132 b}$, T. Akdogan ${ }^{18 a}$, T.P.A. Åkesson ${ }^{78}$, G. Akimoto ${ }^{154}$, A.V. Akimov ${ }^{93}$, M.S. Alam ${ }^{1}$, M.A. Alam ${ }^{75}$, J. Albert $^{168}$, S. Albrand ${ }^{54}$, M. Aleksa ${ }^{29}$, I.N. Aleksandrov ${ }^{63}$, F. Alessandria ${ }^{88 a}$, C. Alexa ${ }^{25 a}$, G. Alexander ${ }^{152}$, G. Alexan$\mathrm{dre}^{48}$, T. Alexopoulos ${ }^{9}$, M. Alhroob ${ }^{163 a, 163 c}$, M. Aliev ${ }^{15}$, G. Alimonti ${ }^{88 a}$, J. Alison ${ }^{119}$, B.M.M. Allbrooke ${ }^{17}$, P.P. Allport ${ }^{72}$, S.E. Allwood-Spiers ${ }^{52}$, J. Almond ${ }^{81}$, A. Aloisio ${ }^{101 a, 101 b}$, R. Alon ${ }^{171}$, A. Alonso ${ }^{78}$, F. Alonso ${ }^{69}$, B. Alvarez Gonzalez ${ }^{87}$, M.G. Alviggi ${ }^{101 a, 101 b}$, K. Amako ${ }^{64}$, C. Amelung ${ }^{22}$, V.V. Ammosov ${ }^{127, *}$, A. Amorim ${ }^{123 a, b}$, N. Amram ${ }^{152}$, C. Anastopoulos ${ }^{29}$, L.S. Ancu ${ }^{16}$, N. Andari ${ }^{114}$, T. Andeen ${ }^{34}$, C.F. Anders ${ }^{57 b}$, G. Anders ${ }^{57 a}$, K.J. Anderson ${ }^{30}$, A. Andreazza ${ }^{88 a, 88 b}$, V. Andrei ${ }^{57 a}$, M-L. Andrieux ${ }^{54}$, X.S. Anduaga ${ }^{69}$, P. Anger ${ }^{43}$, A. Angerami ${ }^{34}$, F. Anghinolfi ${ }^{29}$, A. Anisenkov ${ }^{106}$, N. Anjos ${ }^{123 a}$, A. Annovi $^{46}$, A. Antonaki ${ }^{8}$, M. Antonelli ${ }^{46}$, A. Antonov ${ }^{95}$, J. Antos ${ }^{143 b}$, F. Anulli ${ }^{131 a}$, M. Aoki ${ }^{100}$, S. Aoun ${ }^{82}$, L. Aperio Bella ${ }^{4}$, R. Apolle ${ }^{117, \mathrm{c}}$, G. Arabidze ${ }^{87}$, I. Aracena ${ }^{142}$, Y. Arai ${ }^{64}$, A.T.H. Arce ${ }^{44}$, S. Arfaouii ${ }^{147}$, J-F. Arguin ${ }^{14}$, E. Arik ${ }^{18 a, *}$, M. Arik ${ }^{18 a}$, A.J. Armbruster ${ }^{86}$, O. Arnaez ${ }^{80}$, V. Arnal ${ }^{79}$, C. Arnault ${ }^{114}$, A. Artamonov ${ }^{94}$, G. Artoni ${ }^{131 a, 131 b}$, D. Arutinov ${ }^{20}$, S. Asai ${ }^{154}$, R. Asfandiyarov ${ }^{172}$, S. Ask ${ }^{27}$, B. Åsman ${ }^{145 a, 145 b}$, L. Asquith ${ }^{5}$, K. Assamagann ${ }^{24}$, A. Astbury ${ }^{168}$, B. Aubert ${ }^{4}$, E. Auge ${ }^{114}$, K. Augsten ${ }^{126}$, M. Aurousseau ${ }^{144 a}$, G. Avolio ${ }^{162}$, R. Avramidou ${ }^{9}$, D. Axen ${ }^{167}$, G. Azuelos ${ }^{92, d}$, Y. Azuma ${ }^{154}$, M.A. Baak ${ }^{29}$, G. Baccaglioni ${ }^{88 a}$, C. Bacci 133a,133b, A.M. Bach ${ }^{14}$, H. Bachacou ${ }^{135}$, K. Bachas ${ }^{29}$, M. Backes ${ }^{48}$, M. Backhaus ${ }^{20}$, E. Badescu $^{25 a}$, P. Bagnaia ${ }^{131 a, 131 b}$, S. Bahinipati ${ }^{2}$, Y. Bai ${ }^{32 a}$, D.C. Bailey ${ }^{157}$, T. Bain ${ }^{157}$, J.T. Baines ${ }^{128}$, O.K. Baker ${ }^{175}$, M.D. Baker ${ }^{24}$, S. Baker ${ }^{76}$, E. Banas ${ }^{38}$, P. Banerjee ${ }^{92}$, Sw. Banerjee ${ }^{172}$, D. Banfi ${ }^{29}$, A. Bangert ${ }^{149}$, V. Bansal ${ }^{168}$, H.S. Bansil ${ }^{17}$, L. Barak ${ }^{171}$,

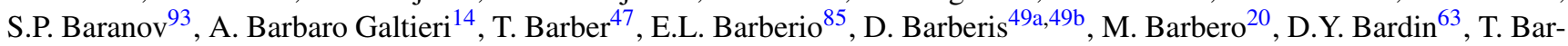
illari $^{98}$, M. Barisonzi ${ }^{174}$, T. Barklow ${ }^{142}$, N. Barlow ${ }^{27}$, B.M. Barnett ${ }^{128}$, R.M. Barnett ${ }^{14}$, A. Baroncelli ${ }^{133 a}$, G. Barone ${ }^{48}$, A.J. Barr ${ }^{117}$, F. Barreiro ${ }^{79}$, J. Barreiro Guimarães da Costa $^{56}$, P. Barrillon ${ }^{114}$, R. Bartoldus ${ }^{142}$, A.E. Barton ${ }^{70}$, V. Bartsch ${ }^{148}$, R.L. Bates ${ }^{52}$, L. Batkova ${ }^{143 a}$, J.R. Batley ${ }^{27}$, A. Battaglia ${ }^{16}$, M. Battistin ${ }^{29}$, F. Bauer ${ }^{135}$, H.S. Bawa ${ }^{142, e}$, S. Beale ${ }^{97}$, T. Beau ${ }^{77}$, P.H. Beauchemin ${ }^{160}$, R. Beccherle ${ }^{49 a}$, P. Bechtle ${ }^{20}$, H.P. Beck ${ }^{16}$, A.K. Becker ${ }^{174}$, S. Becker ${ }^{97}$, M. Beckingham ${ }^{137}$, K.H. Becks ${ }^{174}$, A.J. Beddall ${ }^{18 c}$, A. Beddall ${ }^{18 c}$, S. Bedikian ${ }^{175}$, V.A. Bednyakov ${ }^{63}$, C.P. Bee ${ }^{82}$, L.J. Beemster ${ }^{104}$, M. Begel ${ }^{24}$, S. Behar Harpaz ${ }^{151}$, P.K. Behera ${ }^{61}$, M. Beimforde ${ }^{98}$, C. Belanger-Champagne ${ }^{84}$, P.J. Bell ${ }^{48}$, W.H. Bell ${ }^{48}$, G. Bella ${ }^{152}$, L. Bellagamba $^{19 a}$, F. Bellina ${ }^{29}$, M. Bellomo ${ }^{29}$, A. Belloni ${ }^{56}$, O. Beloborodova ${ }^{106, f}$, K. Belotskiy ${ }^{95}$, O. Beltramello ${ }^{29}$, O. Benary ${ }^{152}$, D. Benchekroun ${ }^{134 a}$, K. Bendtz ${ }^{145 a, 145 b}$, N. Benekos ${ }^{164}$, Y. Benhammou ${ }^{152}$, E. Benhar Noccioli ${ }^{48}$, J.A. Benitez Garcia ${ }^{158 b}$, D.P. Benjamin ${ }^{44}$, M. Benoit ${ }^{114}$, J.R. Bensinger ${ }^{22}$, K. Benslama ${ }^{129}$, S. Bentvelsen ${ }^{104}$, D. Berge ${ }^{29}$, E. Bergeaas Kuutmann ${ }^{41}$, N. Berger ${ }^{4}$, F. Berghaus ${ }^{168}$, E. Berglund ${ }^{104}$, J. Beringer ${ }^{14}$, P. Bernat ${ }^{76}$, R. Bernhard ${ }^{47}$, C. Bernius ${ }^{24}$, T. Berry ${ }^{75}$, C. Bertella ${ }^{82}$, A. Bertin ${ }^{19 a, 19 b}$, F. Bertolucci ${ }^{121 a, 121 b}$, M.I. Besana ${ }^{88 a, 88 b}$, G.J. Besjes ${ }^{103}$, N. Besson ${ }^{135}$, S. Bethke ${ }^{98}$, W. Bhimji ${ }^{45}$, R.M. Bianchi ${ }^{29}$, M. Bianco ${ }^{71 \mathrm{a}, 71 \mathrm{~b}}$, O. Biebel ${ }^{97}$, S.P. Bieniek ${ }^{76}$, K. Bierwagen ${ }^{53}$, J. Biesiada ${ }^{14}$, M. Biglietti ${ }^{133 a}$, H. Bilokon ${ }^{46}$, M. Bindi ${ }^{19 a, 19 b}$, S. Binet ${ }^{114}$, A. Bingul ${ }^{18 c}$, C. Bini ${ }^{131 a, 131 b}$, C. Biscarat ${ }^{177}$, U. Bitenc ${ }^{47}$, K.M. Black ${ }^{21}$, R.E. Blair ${ }^{5}$, J.-B. Blanchard $^{135}$, G. Blanchot ${ }^{29}$, T. Blazek ${ }^{143 a}$, C. Blocker ${ }^{22}$, J. Blocki ${ }^{38}$, A. Blondel ${ }^{48}$, W. Blum ${ }^{80}$, U. Blumenschein ${ }^{53}$, G.J. Bobbink $^{104}$, V.B. Bobrovnikov ${ }^{106}$, S.S. Bocchetta ${ }^{78}$, A. Bocci ${ }^{44}$, C.R. Boddy ${ }^{117}$, M. Boehler ${ }^{47}$, J. Boek ${ }^{174}$, N. Boelaert ${ }^{35}$, J.A. Bogaerts ${ }^{29}$, A. Bogdanchikov ${ }^{106}$, A. Bogouch ${ }^{89, *}$, C. Bohm ${ }^{145 a}$, J. Bohm ${ }^{124}$, V. Boisvert ${ }^{75}$, T. Bold ${ }^{37}$, V. Boldea ${ }^{25 a}$, N.M. Bolnet ${ }^{135}$, M. Bomben ${ }^{77}$, M. Bona ${ }^{74}$, M. Boonekamp ${ }^{135}$, C.N. Booth ${ }^{138}$, S. Bordoni ${ }^{77}$, C. Borer ${ }^{16}$, A. Borisov ${ }^{127}$, G. Borissov $^{70}$, I. Borjanovic ${ }^{12 a}$, M. Borri ${ }^{81}$, S. Borroni ${ }^{86}$, V. Bortolotto ${ }^{133 a, 133 b}$, K. Bos ${ }^{104}$, D. Boscherini ${ }^{19 a}$, M. Bosman ${ }^{11}$, H. Boterenbrood ${ }^{104}$, J. Bouchami ${ }^{92}$, J. Boudreau ${ }^{122}$, E.V. Bouhova-Thacker ${ }^{70}$, D. Boumediene ${ }^{33}$, C. Bourdarios ${ }^{114}$, N. Bousson $^{82}$, A. Boveia ${ }^{30}$, J. Boyd ${ }^{29}$, I.R. Boyko ${ }^{63}$, I. Bozovic-Jelisavcic ${ }^{12 b}$, J. Bracinik ${ }^{17}$, P. Branchini ${ }^{133 a}$, G.W. Brandenburg ${ }^{56}$, A. Brandt ${ }^{7}$, G. Brandt ${ }^{117}$, O. Brandt ${ }^{53}$, U. Bratzler ${ }^{155}$, B. Brau ${ }^{83}$, J.E. Brau ${ }^{113}$, H.M. Braun ${ }^{174, *}$, S.F. Brazzale ${ }^{163 a, 163 c}$, B. Brelier ${ }^{157}$, J. Bremer ${ }^{29}$, K. Brendlinger ${ }^{119}$, R. Brenner ${ }^{165}$, S. Bressler ${ }^{171}$, D. Britton ${ }^{52}$, F.M. Brochu ${ }^{27}$, I. Brock ${ }^{20}$, R. Brock ${ }^{87}$, F. Broggi ${ }^{88 a}$, C. Bromberg ${ }^{87}$, J. Bronner ${ }^{98}$, G. Brooijmans ${ }^{34}$, T. Brooks ${ }^{75}$, W.K. Brooks ${ }^{31 b}$, G. Brown ${ }^{81}$,

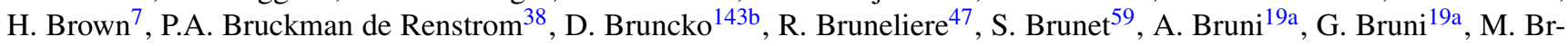
uschi $^{19 a}$, T. Buanes ${ }^{13}$, Q. Buat ${ }^{54}$, F. Bucci ${ }^{48}$, J. Buchanan ${ }^{117}$, P. Buchholz ${ }^{140}$, R.M. Buckingham ${ }^{117}$, A.G. Buckley ${ }^{45}$, S.I. Buda ${ }^{25 a}$, I.A. Budagov ${ }^{63}$, B. Budick ${ }^{107}$, V. Büscher ${ }^{80}$, L. Bugge ${ }^{116}$, O. Bulekov ${ }^{95}$, A.C. Bundock ${ }^{72}$, M. Bunse ${ }^{42}$, T. Buran ${ }^{116}$, H. Burckhart ${ }^{29}$, S. Burdin ${ }^{72}$, T. Burgess ${ }^{13}$, S. Burke ${ }^{128}$, E. Busato ${ }^{33}$, P. Bussey ${ }^{52}$, C.P. Buszello ${ }^{165}$, B. Butler $^{142}$, J.M. Butler ${ }^{21}$, C.M. Buttar ${ }^{52}$, J.M. Butterworth ${ }^{76}$, W. Buttinger ${ }^{27}$, S. Cabrera Urbán ${ }^{166}$, D. Caforio ${ }^{19 a, 19 b}$, O. Cakir ${ }^{3 a}$, P. Calafiura ${ }^{14}$, G. Calderini ${ }^{77}$, P. Calfayan ${ }^{97}$, R. Calkins ${ }^{105}$, L.P. Caloba ${ }^{23 a}$, R. Caloi ${ }^{131 a, 131 b}$, D. Calvet ${ }^{33}$, S. Calvet ${ }^{33}$, R. Camacho Toro ${ }^{33}$, P. Camarri1 ${ }^{132 a}$,132b, D. Cameron ${ }^{116}$, L.M. Caminada ${ }^{14}$, S. Campana ${ }^{29}$, M. Campanelli ${ }^{76}$, V. Canale ${ }^{101 a, 101 b}$, F. Canelli ${ }^{30, g}$, A. Canepa ${ }^{158 a}$, J. Cantero ${ }^{79}$, R. Cantrill ${ }^{75}$, L. Capasso ${ }^{101 a, 101 b}$, M.D.M. Capeans Garrido ${ }^{29}$, I. Caprini ${ }^{25 a}$, 
M. Caprinii ${ }^{25 a}$, D. Capriotti ${ }^{98}$, M. Capua ${ }^{36 a, 36 b}$, R. Caputo $^{80}$, R. Cardarelli ${ }^{132 a}$, T. Carli ${ }^{29}$, G. Carlino ${ }^{101 a}$, L. Carminati $^{88 a, 88 b}$, B. Caron ${ }^{84}$, S. Caron ${ }^{103}$, E. Carquin ${ }^{31 b}$, G.D. Carrillo Montoya ${ }^{172}$, A.A. Carter ${ }^{74}$, J.R. Carter ${ }^{27}$, J. Carvalho ${ }^{123 a, h}$, D. Casadei ${ }^{107}$, M.P. Casado ${ }^{11}$, M. Cascella ${ }^{121 a, 121 b}$, C. Caso 49a,49b,* A.M. Castaneda Hernandez ${ }^{172, i}$, E. CastanedaMiranda $^{172}$, V. Castillo Gimenez ${ }^{166}$, N.F. Castro ${ }^{123 a}$, G. Cataldi ${ }^{71 a}$, P. Catastini ${ }^{56}$, A. Catinaccio ${ }^{29}$, J.R. Catmore ${ }^{29}$, A. Cattai $^{29}$, G. Cattani ${ }^{132 a, 132 b}$, S. Caughron ${ }^{87}$, P. Cavalleri ${ }^{77}$, D. Cavalli ${ }^{88 a}$, M. Cavalli-Sforza ${ }^{11}$, V. Cavasinni121a,121b ${ }^{12}$ F. Ceradini $^{133 a, 133 b}$, A.S. Cerqueira ${ }^{23 b}$, A. Cerri ${ }^{29}$, L. Cerrito $^{74}$, F. Cerutti ${ }^{46}$, S.A. Cetin ${ }^{18 b}$, A. Chafaq ${ }^{134 a}$, D. Chakraborty ${ }^{105}$, I. Chalupkova ${ }^{125}$, K. Chan $^{2}$, B. Chapleau ${ }^{84}$, J.D. Chapmann ${ }^{27}$, J.W. Chapman ${ }^{86}$, E. Chareyre ${ }^{77}$, D.G. Charlton ${ }^{17}$, V. Chavda ${ }^{81}$, C.A. Chavez Barajas ${ }^{29}$, S. Cheatham ${ }^{84}$, S. Chekanov ${ }^{5}$, S.V. Chekulaev ${ }^{158 a}$, G.A. Chelkov ${ }^{63}$, M.A. Chelstowska ${ }^{103}$, C. Chen ${ }^{62}$, H. Chen ${ }^{24}$, S. Chen ${ }^{32 c}$, X. Chen ${ }^{172}$, Y. Chen ${ }^{34}$, A. Cheplakov ${ }^{63}$, R. Cherkaoui El Moursli ${ }^{134 e}$, V. Chernyatin $^{24}$, E. Cheu ${ }^{6}$, S.L. Cheung ${ }^{157}$, L. Chevalier ${ }^{135}$, G. Chiefari ${ }^{101 a, 101 b}$, L. Chikovani ${ }^{50 a, *}$, J.T. Childers ${ }^{29}$, A. Chilingarov ${ }^{70}$, G. Chiodini ${ }^{71 a}$, A.S. Chisholm ${ }^{17}$, R.T. Chislett ${ }^{76}$, A. Chitan ${ }^{25 a}$, M.V. Chizhov ${ }^{63}$, G. Choudalakis ${ }^{30}$, S. Chouridou ${ }^{136}$, I.A. Christidi ${ }^{76}$, A. Christov $^{47}$, D. Chromek-Burckhart ${ }^{29}$, M.L. Chu ${ }^{150}$, J. Chudoba ${ }^{124}$, G. Ciapetti ${ }^{131 a, 131 b}$, A.K. Ciftci ${ }^{3 a}$, R. Ciftci ${ }^{3 a}$, D. Cinca ${ }^{33}$, V. Cindro ${ }^{73}$, C. Ciocca ${ }^{19 a, 19 b}$, A. Ciocio ${ }^{14}$, M. Cirilli ${ }^{86}$, P. Cirkovic ${ }^{12 b}$, M. Citterio ${ }^{88 a}$, M. Ciubancann $^{25 a}$, A. Clark ${ }^{48}$, P.J. Clark ${ }^{45}$, R.N. Clarke ${ }^{14}$, W. Cleland ${ }^{122}$, J.C. Clemens ${ }^{82}$, B. Clement ${ }^{54}$, C. Clement ${ }^{145 a, 145 b}$, Y. Coadou ${ }^{82}$, M. Cobal ${ }^{163 a}, 163 c$, A. Coccaro ${ }^{137}$, J. Cochran ${ }^{62}$, J.G. Cogan ${ }^{142}$, J. Coggeshall ${ }^{164}$, E. Cogneras ${ }^{177}$, J. Colas $^{4}$, S. Cole ${ }^{105}$, A.P. Colijn ${ }^{104}$, N.J. Collins ${ }^{17}$, C. Collins-Tooth ${ }^{52}$, J. Collot ${ }^{54}$, T. Colombo ${ }^{118 a, 118 b}$, G. Colon ${ }^{83}$, P. Conde Muiño $^{123 a}$, E. Coniavitis ${ }^{117}$, M.C. Conidi ${ }^{11}$, S.M. Consonni ${ }^{88 a, 88 b}$, V. Consorti ${ }^{47}$, S. Constantinescu ${ }^{25 a}$, C. Conta ${ }^{118 a, 118 b}$, G. Conti ${ }^{56}$, F. Conventi ${ }^{101 a, j}$, M. Cooke ${ }^{14}$, B.D. Cooper $^{76}$, A.M. Cooper-Sarkar ${ }^{117}$, K. Copic ${ }^{14}$, T. Cornelissen ${ }^{174}$, M. Corradi $^{19 a}$, F. Corriveau ${ }^{84, k}$, A. Cortes-Gonzalez ${ }^{164}$, G. Cortiana ${ }^{98}$, G. Costa $^{88 a}$, M.J. Costa ${ }^{166}$, D. Costanzo ${ }^{138}$, T. Costin ${ }^{30}$, D. Côté ${ }^{29}$, L. Courneyea ${ }^{168}$, G. Cowan ${ }^{75}$, C. Cowden $^{27}$, B.E. Cox $^{81}$, K. Cranmer ${ }^{107}$, F. Crescioli ${ }^{121 a, 121 b}$, M. Cristinziani ${ }^{20}$, G. Crosetti ${ }^{36 a, 36 b}$, S. Crépé-Renaudin ${ }^{54}$, C.-M. Cuciuc ${ }^{25 a}$, C. Cuenca Almenar ${ }^{175}$, T. Cuhadar Donszelmann ${ }^{138}$, M. Curatolo $^{46}$, C.J. Curtis ${ }^{17}$, C. Cuthbert ${ }^{149}$, P. Cwetanski ${ }^{59}$, H. Czirr ${ }^{140}$, P. Czodrowski ${ }^{43}$, Z. Czyczula ${ }^{175}$, S. D'Auria ${ }^{52}$, M. D’Onofrio ${ }^{72}$, A. D’Orazio ${ }^{131 a, 131 b}$, M.J. Da Cunha Sargedas De Sousa ${ }^{123 a}$, C. Da Via ${ }^{81}$, W. Dabrowski ${ }^{37}$, A. Dafinca ${ }^{117}$, T. Dai ${ }^{86}$, C. Dallapiccola ${ }^{83}$, M. Dam ${ }^{35}$, M. Dameri ${ }^{49 a} 49 \mathrm{~b}$, D.S. Damiani ${ }^{136}$, H.O. Danielsson ${ }^{29}$, V. Dao ${ }^{48}$, G. Darbo $^{49 a}$, G.L. Darlea ${ }^{25 b}$, J.A. Dassoulas ${ }^{41}$, W. Davey ${ }^{20}$, T. Davidek ${ }^{125}$, N. Davidson ${ }^{85}$, R. Davidson ${ }^{70}$, E. Davies ${ }^{117, c}$, M. Davies ${ }^{92}$, O. Davignon ${ }^{77}$, A.R. Davison ${ }^{76}$, Y. Davygora ${ }^{57 a}$, E. Dawe ${ }^{141}$, I. Dawson ${ }^{138}$, R.K. Daya-Ishmukhametova ${ }^{22}$, K. De ${ }^{7}$, R. de Asmundis ${ }^{101 a}$, S. De Castro ${ }^{19 a}$,19b, S. De Cecco ${ }^{77}$, J. de Graat ${ }^{97}$, N. De Groot ${ }^{103}$, P. de Jong ${ }^{104}$, C. De La Taille ${ }^{114}$, H. De la Torre ${ }^{79}$, F. De Lorenzi ${ }^{62}$, L. de Mora $^{70}$, L. De Nooij ${ }^{104}$, D. De Pedis ${ }^{131 a}$, A. De Salvo ${ }^{131 a}$, U. De Sanctis $^{163 a, 163 c}$, A. De Santo ${ }^{148}$, J.B. De Vivie De Regie ${ }^{114}$, G. De Zorzi ${ }^{131 a, 131 b}$, W.J. Dearnaley ${ }^{70}$, R. Debbe ${ }^{24}$, C. Debenedetti ${ }^{45}$, B. Dechenaux ${ }^{54}$, D.V. Dedovich ${ }^{63}$, J. Degenhardt ${ }^{119}$, C. Del Papa ${ }^{163 a, 163 c}$, J. Del Peso ${ }^{79}$, T. Del Prete ${ }^{121 a, 121 b}$, T. Delemonte $^{54}$, M. Deliyergiyev ${ }^{73}$, A. Dell'Acqua ${ }^{29}$, L. Dell'Asta ${ }^{21}$, M. Della Pietra ${ }^{101 a, j}$, D. della Volpe ${ }^{101 a, 101 b}$, M. Delmastro $^{4}$, P.A. Delsart ${ }^{54}$, C. Deluca ${ }^{104}$, S. Demers ${ }^{175}$, M. Demichev ${ }^{63}$, B. Demirkoz ${ }^{11,1}$, J. Deng ${ }^{162}$, S.P. Denisov ${ }^{127}$, D. Derendarz ${ }^{38}$, J.E. Derkaoui ${ }^{134 d}$, F. Derue ${ }^{77}$, P. Dervan ${ }^{72}$, K. Desch $^{20}$, E. Devetak ${ }^{147}$, P.O. Deviveiros ${ }^{104}$, A. Dewhurst ${ }^{128}$, B. DeWilde ${ }^{147}$, S. Dhaliwal ${ }^{157}$, R. Dhullipudi ${ }^{24, m}$, A. Di Ciaccio ${ }^{132 a, 132 b}$, L. Di Ciaccio ${ }^{4}$, A. Di Girolamo ${ }^{29}$, B. Di Girolamo $^{29}$, S. Di Luise ${ }^{133 a, 133 b}$, A. Di Mattia ${ }^{172}$, B. Di Micco ${ }^{29}$, R. Di Nardo ${ }^{46}$, A. Di Simone ${ }^{132 a, 132 b}$, R. Di Sipio ${ }^{19 a, 19 b}$, M.A. Diaz ${ }^{31 a}$, E.B. Diehl ${ }^{86}$, J. Dietrich ${ }^{41}$, T.A. Dietzsch ${ }^{57 a}$, S. Diglio ${ }^{85}$, K. Dindar Yagci $^{39}$, J. Dingfelder ${ }^{20}$, F. Dinut ${ }^{25 a}$, C. Dionisi ${ }^{131 a, 131 b}$, P. Dita ${ }^{25 a}$, S. Dita ${ }^{25 a}$, F. Dittus ${ }^{29}$, F. Djama ${ }^{82}$, T. Djobava ${ }^{50 b}$, M.A.B. do Vale ${ }^{23 c}$, A. Do Valle Wemans $^{123 a, n}$, T.K.O. Doan ${ }^{4}$, M. Dobbs ${ }^{84}$, R. Dobinson ${ }^{29, *}$, D. Dobos ${ }^{29}$, E. Dobson ${ }^{29, o}$, J. Dodd $^{34}$, C. Doglioni ${ }^{48}$, T. Doherty ${ }^{52}$, Y. Doi ${ }^{64, *}$, J. Dolejsi ${ }^{125}$, I. Dolenc ${ }^{73}$, Z. Dolezal ${ }^{125}$, B.A. Dolgoshein ${ }^{95, *}$, T. Dohmae ${ }^{154}$, M. Donadelli ${ }^{23 d}$, J. Donini ${ }^{33}$, J. Dopke ${ }^{29}$, A. Doria ${ }^{101 a}$, A. Dos Anjos $^{172}$, A. Dotti ${ }^{121 a, 121 b}$, M.T. Dova ${ }^{69}$, A.D. Doxiadis ${ }^{104}$, A.T. Doyle ${ }^{52}$, N. Dressnandt $^{119}$, M. Dris ${ }^{9}$, J. Dubbert ${ }^{98}$, S. Dube ${ }^{14}$, E. Duchovni1 ${ }^{171}$, G. Duckeck ${ }^{97}$, A. Dudarev ${ }^{29}$, F. Dudziak ${ }^{62}$, M. Dührssen ${ }^{29}$,

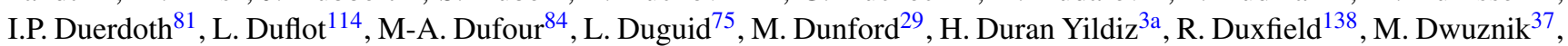
F. Dydak ${ }^{29}$, M. Düren ${ }^{51}$, W.L. Ebenstein ${ }^{44}$, J. Ebke ${ }^{97}$, S. Eckweiler $^{80}$, K. Edmonds ${ }^{80}$, W. Edson ${ }^{1}$, C.A. Edwards ${ }^{75}$, N.C. Edwards $^{52}$, W. Ehrenfeld ${ }^{41}$, T. Eifert ${ }^{142}$, G. Eigen ${ }^{13}$, K. Einsweiler ${ }^{14}$, E. Eisenhandler ${ }^{74}$, T. Ekelof ${ }^{165}$, M. El Kacimi ${ }^{134 c}$, M. Ellert ${ }^{165}$, S. Elles $^{4}$, F. Ellinghaus ${ }^{80}$, K. Ellis $^{74}$, N. Ellis $^{29}$, J. Elmsheuser ${ }^{97}$, M. Elsing ${ }^{29}$, D. Emeliyanov ${ }^{128}$, R. Engelmann $^{147}$, A. Engl ${ }^{97}$, B. Epp ${ }^{60}$, J. Erdmann ${ }^{53}$, A. Ereditato ${ }^{16}$, D. Eriksson ${ }^{145 a}$, J. Ernst ${ }^{1}$, M. Ernst ${ }^{24}$, J. Ernwein ${ }^{135}$, D. Errede ${ }^{164}$, S. Errede ${ }^{164}$, E. Ertel ${ }^{80}$, M. Escalier ${ }^{114}$, H. Esch ${ }^{42}$, C. Escobar ${ }^{122}$, X. Espinal Curull ${ }^{11}$, B. Esposito ${ }^{46}$, F. Etienne ${ }^{82}$, A.I. Etienvre ${ }^{135}$, E. Etzion ${ }^{152}$, D. Evangelakou ${ }^{53}$, H. Evans ${ }^{59}$, L. Fabbri ${ }^{19 a, 19 b}$, C. Fabre ${ }^{29}$, R.M. Fakhrutdinov $^{127}$, S. Falciano ${ }^{131 a}$, Y. Fang ${ }^{172}$, M. Fanti ${ }^{88,88 b}$, A. Farbin ${ }^{7}$, A. Farilla ${ }^{133 a}$, J. Farley ${ }^{147}$, T. Farooque ${ }^{157}$, S. Farrell ${ }^{162}$, S.M. Farrington ${ }^{169}$, P. Farthouat ${ }^{29}$, P. Fassnacht ${ }^{29}$, D. Fassouliotis ${ }^{8}$, B. Fatholahzadeh ${ }^{157}$, A. Favareto ${ }^{88 a, 88 b}$, L. Fayard ${ }^{114}$, S. Fazio $^{36 a, 36 b}$, R. Febbraro ${ }^{33}$, P. Federic ${ }^{143 a}$, O.L. Fedin ${ }^{120}$, W. Fedorko ${ }^{87}$, M. Fehling-Kaschek ${ }^{47}$, L. Feligioni ${ }^{82}$, D. Fellmann $^{5}$, C. Feng ${ }^{32 d}$, E.J. Feng ${ }^{5}$, A.B. Fenyuk ${ }^{127}$, J. Ferencei ${ }^{143 b}$, W. Fernando ${ }^{5}$, S. Ferrag ${ }^{52}$, J. Ferrando ${ }^{52}$, V. Ferrara ${ }^{41}$, A. Ferrari ${ }^{165}$, P. Ferrari ${ }^{104}$, R. Ferrari ${ }^{18 a}$, D.E. Ferreira de Lima $^{52}$, A. Ferrer ${ }^{166}$, D. Ferrere ${ }^{48}$, C. Ferretti ${ }^{86}$, A. Ferretto Parodi ${ }^{49 a, 49 b}$, M. Fiascaris ${ }^{30}$, F. Fiedler ${ }^{80}$, A. Filipčič $\check{c}^{73}$, F. Filthaut ${ }^{103}$, M. Fincke-Keeler ${ }^{168}$, M.C.N. Fiolhais ${ }^{123 a, h}$, 
L. Fiorini ${ }^{166}$, A. Firan ${ }^{39}$, G. Fischer ${ }^{41}$, M.J. Fisher ${ }^{108}$, M. Flechl ${ }^{47}$, I. Fleck ${ }^{140}$, J. Fleckner ${ }^{80}$, P. Fleischmann ${ }^{173}$, S. Fleischmann $^{174}$, T. Flick ${ }^{174}$, A. Floderus ${ }^{78}$, L.R. Flores Castillo ${ }^{172}$, M.J. Flowerdew ${ }^{98}$, T. Fonseca Martin ${ }^{16}$, A. Formica ${ }^{135}$, A. Forti ${ }^{81}$, D. Fortin ${ }^{158 a}$, D. Fournier ${ }^{114}$, A.J. Fowler ${ }^{44}$, H. Fox $^{70}$, P. Francavilla ${ }^{11}$, M. Franchini ${ }^{19 a, 19 b}$, S. Franchino ${ }^{118 a, 118 b}$, D. Francis ${ }^{29}$, T. Frank ${ }^{171}$, S. Franz ${ }^{29}$, M. Fraternali118a,118b , S. Fratina ${ }^{119}$, S.T. French ${ }^{27}$, C. Friedrich ${ }^{41}$, F. Friedrich ${ }^{43}$, R. Froeschl ${ }^{29}$, D. Froidevaux ${ }^{29}$, J.A. Frost ${ }^{27}$, C. Fukunaga ${ }^{155}$, E. Fullana Torregrosa ${ }^{29}$, B.G. Fulsom ${ }^{142}$, J. Fuster ${ }^{166}$, C. Gabaldon ${ }^{29}$, O. Gabizon ${ }^{171}$, T. Gadfort ${ }^{24}$, S. Gadomski ${ }^{48}$, G. Gagliardi49a,49b ${ }^{49}$, Gagnon ${ }^{59}$, C. Galea ${ }^{97}$, E.J. Gallas $^{117}$, V. Gallo ${ }^{16}$, B.J. Gallop ${ }^{128}$, P. Gallus ${ }^{124}$, K.K. Gan ${ }^{108}$, Y.S. Gao ${ }^{142, \text { e }}$, A. Gaponenko ${ }^{14}$, F. Garberson ${ }^{175}$, M. GarciaSciveres $^{14}$, C. García ${ }^{166}$, J.E. García Navarro ${ }^{166}$, R.W. Gardner ${ }^{30}$, N. Garellii ${ }^{29}$, H. Garitaonandia ${ }^{104}$, V. Garonne ${ }^{29}$, J. Gar$v y^{17}$, C. Gatti ${ }^{46}$, G. Gaudio ${ }^{118 a}$, B. Gaur ${ }^{140}$, L. Gauthier ${ }^{135}$, P. Gauzzi ${ }^{131 a, 131 b}$, I.L. Gavrilenko ${ }^{93}$, C. Gay ${ }^{167}$, G. Gaycken $^{20}$, E.N. Gazis ${ }^{9}$, P. Ge ${ }^{32 d}$, Z. Gecse ${ }^{167}$, C.N.P. Gee ${ }^{128}$, D.A.A. Geerts ${ }^{104}$, Ch. Geich-Gimbel ${ }^{20}$, K. Gellerstedt ${ }^{145 a, 145 b}$, C. Gemme ${ }^{49 a}$, A. Gemmell ${ }^{52}$, M.H. Genest ${ }^{54}$, S. Gentile ${ }^{131 a, 131 b}$, M. George ${ }^{53}$, S. George ${ }^{75}$, P. Gerlach ${ }^{174}$, A. Gershon $^{152}$, C. Geweniger ${ }^{57 a}$, H. Ghazlane ${ }^{134 b}$, N. Ghodbane ${ }^{33}$, B. Giacobbe ${ }^{19 a}$, S. Giagu 131a,131b, V. Giakoumopoulou ${ }^{8}$, V. Giangiobbe ${ }^{11}$, F. Gianotti ${ }^{29}$, B. Gibbard ${ }^{24}$, A. Gibson ${ }^{157}$, S.M. Gibson ${ }^{29}$, D. Gillberg ${ }^{28}$, A.R. Gillman ${ }^{128}$, D.M. Gingrich $^{2, \mathrm{~d}}$, J. Ginzburg ${ }^{152}$, N. Giokaris ${ }^{8}$, M.P. Giordani ${ }^{163 c}$, R. Giordano ${ }^{101 a, 101 b}$, F.M. Giorgi ${ }^{15}$, P. Giovannini ${ }^{98}$, P.F. Gi-

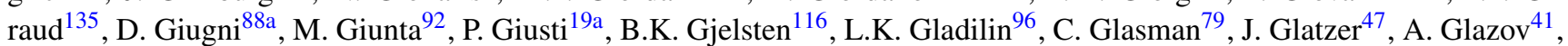
K.W. Glitza ${ }^{174}$, G.L. Glonti ${ }^{63}$, J.R. Goddard ${ }^{74}$, J. Godfrey ${ }^{141}$, J. Godlewski ${ }^{29}$, M. Goebel ${ }^{41}$, T. Göpfert ${ }^{43}$, C. Goeringer ${ }^{80}$, C. Gössling ${ }^{42}$, S. Goldfarb ${ }^{86}$, T. Golling ${ }^{175}$, A. Gomes ${ }^{123 a, b}$, L.S. Gomez Fajardo ${ }^{41}$, R. Gonçalo ${ }^{75}$, J. Goncalves Pinto Firmino Da Costa ${ }^{41}$, L. Gonella ${ }^{20}$, S. Gonzalez ${ }^{172}$, S. González de la $\mathrm{Hoz}^{166}$, G. Gonzalez Parra ${ }^{11}$, M.L. Gonzalez Silva ${ }^{26}$, S. Gonzalez-Sevilla ${ }^{48}$, J.J. Goodson ${ }^{147}$, L. Goossens ${ }^{29}$, P.A. Gorbounov ${ }^{94}$, H.A. Gordon ${ }^{24}$, I. Gorelov ${ }^{102}$, G. Gorfine ${ }^{174}$, B. Gorinii ${ }^{29}$, E. Gorini ${ }^{71 a, 71 b}$, A. Gorišek ${ }^{73}$, E. Gornicki ${ }^{38}$, B. Gosdzik ${ }^{41}$, A.T. Goshaw ${ }^{5}$, M. Gosselink $^{104}$, M.I. Gostkin ${ }^{63}$, I. Gough Eschrich ${ }^{162}$, M. Gouighri ${ }^{134 a}$, D. Goujdami ${ }^{134 c}$, M.P. Goulette ${ }^{48}$, A.G. Goussiou ${ }^{137}$, C. Goy $^{4}$, S. Gozpinar ${ }^{22}$, I. Grabowska-Bold ${ }^{37}$, P. Grafström ${ }^{19 a, 19 b}$, K-J. Grahn ${ }^{41}$, F. Grancagnolo ${ }^{71 a}$, S. Grancagnolo ${ }^{15}$, V. Grassi ${ }^{147}$, V. Gratchev ${ }^{120}$, N. Grau ${ }^{34}$, H.M. Gray ${ }^{29}$, J.A. Gray ${ }^{147}$, E. Graziani ${ }^{133 a}$, O.G. Grebenyuk ${ }^{120}$, T. Greenshaw ${ }^{72}$, Z.D. Greenwood ${ }^{24, m}$, K. Gregersen ${ }^{35}$, I.M. Gregor ${ }^{41}$, P. Grenier ${ }^{142}$, J. Griffiths ${ }^{137}$, N. Grigalashvili63 ${ }^{63}$ A.A. Grillo ${ }^{136}$, S. Grinstein ${ }^{11}$, Y.V. Grishkevich ${ }^{96}$, J.-F. Grivaz ${ }^{114}$, E. Gross ${ }^{171}$, J. Grosse-Knetter ${ }^{53}$, J. Groth-Jensen ${ }^{171}$, K. Grybel ${ }^{140}$, D. Guest ${ }^{175}$, C. Guicheney ${ }^{33}$, S. Guindon ${ }^{53}$, U. Gul ${ }^{52}$, H. Guler ${ }^{84, p}$, J. Gunther ${ }^{124}$, B. Guo ${ }^{157}$, J. Guo ${ }^{34}$, P. Gutierrez ${ }^{110}$, N. Guttman ${ }^{152}$, O. Gutzwiller ${ }^{172}$, C. Guyot ${ }^{135}$, C. Gwenlan ${ }^{117}$, C.B. Gwilliam ${ }^{72}$, A. Haas ${ }^{142}$, S. Haas ${ }^{29}$, C. Haber ${ }^{14}$, H.K. Hadavand ${ }^{39}$, D.R. Hadley ${ }^{17}$, P. Haefner ${ }^{20}$, F. $\operatorname{Hahn}^{29}$, S. Haider ${ }^{29}$, Z. Hajduk ${ }^{38}$, H. Hakobyan ${ }^{176}$, D. Hall ${ }^{117}$, J. Haller ${ }^{53}$, K. Hamacher ${ }^{174}$, P. Hamal ${ }^{112}$, M. Hamer ${ }^{53}$, A. Hamilton ${ }^{144 b, q}$, S. Hamilton ${ }^{160}$, L. Han ${ }^{32 b}$, K. Hanagaki ${ }^{115}$, K. Hanawa ${ }^{159}$, M. Hance ${ }^{14}$, C. Handel ${ }^{80}$, P. Hanke ${ }^{57 a}$, J.R. Hansen ${ }^{35}$, J.B. Hansen ${ }^{35}$, J.D. Hansen ${ }^{35}$, P.H. Hansen ${ }^{35}$, P. Hansson ${ }^{142}$, K. Hara ${ }^{159}$, G.A. Hare ${ }^{136}$, T. Harenberg ${ }^{174}$, S. Harkusha ${ }^{89}$, D. Harper ${ }^{86}$, R.D. Harrington ${ }^{45}$, O.M. Har-

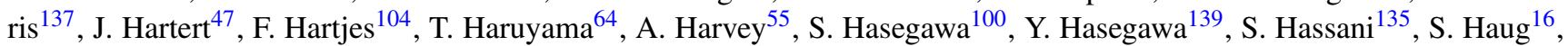
M. Hauschild ${ }^{29}$, R. Hauser ${ }^{87}$, M. Havranek ${ }^{20}$, C.M. Hawkes ${ }^{17}$, R.J. Hawkings ${ }^{29}$, A.D. Hawkins ${ }^{78}$, D. Hawkins ${ }^{162}$, T. Hayakawa ${ }^{65}$, T. Hayashi ${ }^{159}$, D. Hayden ${ }^{75}$, C.P. Hays ${ }^{117}$, H.S. Hayward ${ }^{72}$, S.J. Haywood ${ }^{128}$, M. He ${ }^{32 d}$, S.J. Head ${ }^{17}$, V. Hedberg ${ }^{78}$, L. Heelan ${ }^{7}$, S. Heim ${ }^{87}$, B. Heinemann ${ }^{14}$, S. Heisterkamp ${ }^{35}$, L. Helary ${ }^{21}$, C. Heller ${ }^{97}$, M. Heller ${ }^{29}$, S. Hell$\operatorname{man}^{145 a, 145 b}$, D. Hellmich ${ }^{20}$, C. Helsens ${ }^{11}$, R.C.W. Henderson ${ }^{70}$, M. Henke ${ }^{57 a}$, A. Henrichs ${ }^{53}$, A.M. Henriques Correia ${ }^{29}$, S. Henrot-Versille ${ }^{114}$, C. Hensel ${ }^{53}$, T. Hen $\beta^{174}$, C.M. Hernandez ${ }^{7}$, Y. Hernández Jiménez ${ }^{166}$, R. Herrberg ${ }^{15}$, G. Herten ${ }^{47}$, R. Hertenberger ${ }^{97}$, L. Hervas ${ }^{29}$, G.G. Hesketh ${ }^{76}$, N.P. Hessey ${ }^{104}$, E. Higón-Rodriguez ${ }^{166}$, J.C. Hill ${ }^{27}$, K.H. Hiller ${ }^{41}$, S. Hillert ${ }^{20}$, S.J. Hillier ${ }^{17}$, I. Hinchliffe ${ }^{14}$, E. Hines ${ }^{119}$, M. Hirose ${ }^{115}$, F. Hirsch ${ }^{42}$, D. Hirschbuehl ${ }^{174}$, J. Hobbs ${ }^{147}$, N. Hod ${ }^{152}$, M.C. Hodgkinson ${ }^{138}$, P. Hodgson ${ }^{138}$, A. Hoecker ${ }^{29}$, M.R. Hoeferkamp ${ }^{102}$, J. Hoffman ${ }^{39}$, D. Hoffmann ${ }^{82}$, M. Hohlfeld ${ }^{80}$, M. Holder ${ }^{140}$, S.O. Holmgren ${ }^{145 a}$, T. Holy ${ }^{126}$, J.L. Holzbauer ${ }^{87}$, T.M. Hong ${ }^{119}$, L. Hooft van Huysduynen ${ }^{107}$, C. Horn ${ }^{142}$, S. Horner ${ }^{47}$, J-Y. Hostachy ${ }^{54}$, S. Hou ${ }^{150}$, A. Hoummada ${ }^{134 a}$, J. Howard ${ }^{117}$, J. Howarth ${ }^{81}$, I. Hristova ${ }^{15}$, J. Hrivnac ${ }^{114}$, T. Hryn'ova ${ }^{4}$, P.J. Hsu ${ }^{80}$, S.-C. Hsu ${ }^{14}$, Z. Hubacek ${ }^{126}$, F. Hubaut ${ }^{82}$, F. Huegging ${ }^{20}$, A. Huettmann ${ }^{41}$, T.B. Huffman ${ }^{117}$, E.W. Hughes ${ }^{34}$, G. Hughes ${ }^{70}$, M. Huhtinen ${ }^{29}$, M. Hurwitz ${ }^{14}$, U. Husemann ${ }^{41}$, N. Huseynov ${ }^{63, \mathrm{r}}$, J. Huston ${ }^{87}$, J. Huth ${ }^{56}$, G. Iacobucci ${ }^{48}$, G. Iakovidis ${ }^{9}$, M. Ibbotson ${ }^{81}$, I. Ibragimov ${ }^{140}$, L. Iconomidou-Fayard ${ }^{114}$, J. Idarraga ${ }^{114}$, P. Iengo ${ }^{101 a}$, O. Igonkina ${ }^{104}$, Y. Ikegami ${ }^{64}$, M. Ikeno $^{64}$, D. Iliadis ${ }^{153}$, N. Ilic ${ }^{157}$, T. Ince ${ }^{20}$, J. Inigo-Golfin ${ }^{29}$, P. Ioannou ${ }^{8}$, M. Iodice ${ }^{133 a}$, K. Iordanidou ${ }^{8}$, V. Ippolito ${ }^{131 a, 131 b}$, A. Irles Quiles ${ }^{166}$, C. Isaksson ${ }^{165}$, M. Ishino ${ }^{66}$, M. Ishitsuka ${ }^{156}$, R. Ishmukhameto $^{39}$, C. Issever ${ }^{117}$, S. Istin ${ }^{18 a}$, A.V. Ivashin ${ }^{127}$, W. Iwanski ${ }^{38}$, H. Iwasaki ${ }^{64}$, J.M. Izen ${ }^{40}$, V. Izzo ${ }^{101 a}$, B. Jackson ${ }^{119}$, J.N. Jackson ${ }^{72}$, P. Jackson ${ }^{142}$, M.R. Jaekel ${ }^{29}$, V. Jain ${ }^{59}$, K. Jakobs ${ }^{47}$, S. Jakobsen ${ }^{35}$, T. Jakoubek ${ }^{124}$, J. Jakubek ${ }^{126}$, D.K. Jana ${ }^{110}$, E. Jansen ${ }^{76}$, H. Jansen ${ }^{29}$, A. Jantsch ${ }^{98}$, M. Janus ${ }^{47}$, G. Jarlskog ${ }^{78}$, L. Jeanty ${ }^{56}$, I. Jen-La Plante ${ }^{30}$, D. Jennens $^{85}$, P. Jenni ${ }^{29}$, P. Jež ${ }^{35}$, S. Jézéquel ${ }^{4}$, M.K. Jha ${ }^{19 a}, H_{.} \mathrm{Ji}^{172}$, W. Ji ${ }^{80}$, J. Jia ${ }^{147}$, Y. Jiang ${ }^{32 \mathrm{~b}}$, M. Jimenez Belenguer ${ }^{41}$, S. Jin $^{32 a}$, O. Jinnouchi ${ }^{156}$, M.D. Joergensen ${ }^{35}$, D. Joffe ${ }^{39}$, M. Johansen ${ }^{145 a, 145 b}$, K.E. Johansson ${ }^{145 a}$, P. Johansson ${ }^{138}$, S. Johnert ${ }^{41}$, K.A. Johns ${ }^{6}$, K. Jon-And ${ }^{145 a, 145 b}$, G. Jones ${ }^{169}$, R.W.L. Jones ${ }^{70}$, T.J. Jones ${ }^{72}$, C. Joram ${ }^{29}$, P.M. Jorge ${ }^{123 a}$, 
K.D. Joshi ${ }^{81}$, J. Jovicevic ${ }^{146}$, T. Jovin ${ }^{12 b}$, X. Ju ${ }^{172}$, C.A. Jung ${ }^{42}$, R.M. Jungst ${ }^{29}$, V. Juranek ${ }^{124}$, P. Jussel ${ }^{60}$, A. Juste Rozas $^{11}$, S. Kabana ${ }^{16}$, M. Kaci ${ }^{166}$, A. Kaczmarska ${ }^{38}$, P. Kadlecik ${ }^{35}$, M. Kado ${ }^{114}$, H. Kagan ${ }^{108}$, M. Kagan ${ }^{56}$, E. Kajomovitz $^{151}$, S. Kalinin ${ }^{174}$, L.V. Kalinovskaya ${ }^{63}$, S. Kama ${ }^{39}$, N. Kanaya ${ }^{154}$, M. Kaneda ${ }^{29}$, S. Kaneti ${ }^{27}$, T. Kanno ${ }^{156}$, V.A. Kantserov ${ }^{95}$, J. Kanzaki ${ }^{64}$, B. Kaplan ${ }^{175}$, A. Kapliy ${ }^{30}$, J. Kaplon ${ }^{29}$, D. Kar ${ }^{52}$, M. Karagounis ${ }^{20}$, K. Karakostas ${ }^{9}$, M. Karnevskiy ${ }^{41}$, V. Kartvelishvilii ${ }^{70}$, A.N. Karyukhin ${ }^{127}$, L. Kashif ${ }^{172}$, G. Kasieczka ${ }^{57 b}$, R.D. Kass ${ }^{108}$, A. Kastanas ${ }^{13}$, M. Kataoka ${ }^{4}$, Y. Kataoka ${ }^{154}$, E. Katsoufis ${ }^{9}$, J. Katzy ${ }^{41}$, V. Kaushik ${ }^{6}$, K. Kawagoe ${ }^{68}$, T. Kawamoto ${ }^{154}$, G. Kawamura ${ }^{80}$, M.S. Kayl ${ }^{104}$, V.A. Kazanin ${ }^{106}$, M.Y. Kazarinov ${ }^{63}$, R. Keeler ${ }^{168}$, P.T. Keener ${ }^{119}$, R. Kehoe ${ }^{39}$, M. Keil ${ }^{53}$, G.D. Kekelidze ${ }^{63}$, J.S. Keller ${ }^{137}$, M. Kenyon ${ }^{52}$, O. Kepka ${ }^{124}$, N. Kerschen ${ }^{29}$, B.P. Kerševan ${ }^{73}$, S. Kersten ${ }^{174}$, K. Kessoku ${ }^{154}$, J. Keung ${ }^{157}$, F. Khalil-zada ${ }^{10}$, H. Khandanyan ${ }^{164}$, A. Khanov ${ }^{111}$, D. Kharchenko ${ }^{63}$, A. Khodinov ${ }^{95}$, A. Khomich ${ }^{57 a}$, T.J. Khoo ${ }^{27}$, G. Khoriauli $^{20}$, A. Khoroshilov ${ }^{174}$, V. Khovanskiy ${ }^{94}$, E. Khramov ${ }^{63}$, J. Khubua ${ }^{50 b}$, H. Kim ${ }^{145 a, 145 b}$, S.H. Kim ${ }^{159}$, N. Kimura ${ }^{170}$, O. Kind ${ }^{15}$, B.T. King ${ }^{72}$, M. King ${ }^{65}$, R.S.B. King ${ }^{117}$, J. Kirk ${ }^{128}$, A.E. Kiryunin ${ }^{98}$, T. Kishimoto ${ }^{65}$, D. Kisielewska ${ }^{37}$, T. Kitamura ${ }^{65}$, T. Kittelmann ${ }^{122}$, E. Kladiva ${ }^{143 b}$, M. Klein ${ }^{72}$, U. Klein ${ }^{72}$, K. Kleinknecht ${ }^{80}$, M. Klemetti ${ }^{84}$, A. Klier ${ }^{171}$, P. Klimek ${ }^{145 a, 145 b}$, A. Klimentov ${ }^{24}$, R. Klingenberg ${ }^{42}$, J.A. Klinger ${ }^{81}$, E.B. Klinkby ${ }^{35}$, T. Klioutchnikova ${ }^{29}$, P.F. Klok ${ }^{103}$, S. Klous ${ }^{104}$, E.-E. Kluge ${ }^{57 a}$, T. Kluge ${ }^{72}$, P. Kluit ${ }^{104}$, S. Kluth ${ }^{98}$, N.S. Knecht ${ }^{157}$, E. Kneringer ${ }^{60}$, E.B.F.G. Knoops ${ }^{82}$, A. Knue ${ }^{53}$, B.R. Ko ${ }^{44}$, T. Kobayashi ${ }^{154}$, M. Kobel ${ }^{43}$, M. Kocian ${ }^{142}$, P. Kodys ${ }^{125}$, K. Köneke ${ }^{29}$, A.C. König ${ }^{103}$, S. Koenig ${ }^{80}$, L. Köpke ${ }^{80}$, F. Koetsveld ${ }^{103}$, P. Koevesarki ${ }^{20}$, T. Koffas ${ }^{28}$, E. Koffeman ${ }^{104}$, L.A. Kogan ${ }^{117}$, S. Kohlmann ${ }^{174}$, F. Kohn ${ }^{53}$, Z. Kohout ${ }^{126}$, T. Kohriki ${ }^{64}$, T. Koi ${ }^{142}$, G.M. Kolachev ${ }^{106, *}$, H. Kolanoski ${ }^{15}$, V. Kolesnikov ${ }^{63}$, I. Koletsou ${ }^{88 a}$, J. Koll ${ }^{87}$, M. Kollefrath ${ }^{47}$, A.A. Komar ${ }^{93}$, Y. Komori ${ }^{154}$, T. Kondo ${ }^{64}$, T. Kono ${ }^{41, \mathrm{~s}}$, A.I. Kononov ${ }^{47}$, R. Konoplich ${ }^{107, t}$, N. Konstantinidis $^{76}$, S. Koperny ${ }^{37}$, K. Korcyl ${ }^{38}$, K. Kordas ${ }^{153}$, A. Korn ${ }^{117}$, A. Korol ${ }^{106}$, I. Korolkov ${ }^{11}$, E.V. Korolkova ${ }^{138}$, V.A. Korotkov $^{127}$, O. Kortner ${ }^{98}$, S. Kortner ${ }^{98}$, V.V. Kostyukhin ${ }^{20}$, S. Kotov ${ }^{98}$, V.M. Kotov ${ }^{63}$, A. Kotwal ${ }^{44}$, C. Kourkoumelis ${ }^{8}$, V. Kouskoura ${ }^{153}$, A. Koutsman ${ }^{158 a}$, R. Kowalewski ${ }^{168}$, T.Z. Kowalski ${ }^{37}$, W. Kozanecki ${ }^{135}$, A.S. Kozhin ${ }^{127}$, V. Kral ${ }^{126}$, V.A. Kramarenko ${ }^{96}$, G. Kramberger ${ }^{73}$, M.W. Krasny ${ }^{77}$, A. Krasznahorkay ${ }^{107}$, J.K. Kraus ${ }^{20}$, S. Kreiss ${ }^{107}$, F. Krejci ${ }^{126}$, J. Kretzschmar ${ }^{72}$, N. Krieger ${ }^{53}$, P. Krieger ${ }^{157}$, K. Kroeninger ${ }^{53}$, H. Kroha ${ }^{98}$, J. Kroll ${ }^{119}$, J. Kroseberg ${ }^{20}$, J. Krstic ${ }^{12 a}$, U. Kruchonak ${ }^{63}$, H. Krüger ${ }^{20}$, T. Kruker ${ }^{16}$, N. Krumnack ${ }^{62}$, Z.V. Krumshteyn ${ }^{63}$, T. Kubota ${ }^{85}$, S. Kuday ${ }^{3 a}$, S. Kuehn ${ }^{47}$, A. Kugel ${ }^{57 c}$, T. Kuhl ${ }^{41}$, D. Kuhn ${ }^{60}$, V. Kukhtin ${ }^{63}$, Y. Kulchitsky ${ }^{89}$, S. Kuleshov ${ }^{31 b}$, C. Kummer ${ }^{97}$, M. Kuna ${ }^{77}$, J. Kun$\mathrm{kle}^{119}$, A. Kupco ${ }^{124}$, H. Kurashige ${ }^{65}$, M. Kurata ${ }^{159}$, Y.A. Kurochkin ${ }^{89}$, V. Kus ${ }^{124}$, E.S. Kuwertz ${ }^{146}$, M. Kuze ${ }^{156}$, J. Kvita ${ }^{141}$, R. Kwee ${ }^{15}$, A. La Rosa ${ }^{48}$, L. La Rotonda ${ }^{36 a, 36 b}$, L. Labarga ${ }^{79}$, J. Labbe ${ }^{4}$, S. Lablak ${ }^{134 a}$, C. Lacasta ${ }^{166}$, F. Lacava ${ }^{131 a, 131 b}$, H. Lacker ${ }^{15}$, D. Lacour ${ }^{77}$, V.R. Lacuesta ${ }^{166}$, E. Ladygin ${ }^{63}$, R. Lafaye ${ }^{4}$, B. Laforge ${ }^{77}$, T. Lagouri ${ }^{79}$, S. Lai ${ }^{47}$, E. Laisne ${ }^{54}$, M. Lamanna ${ }^{29}$, L. Lambourne ${ }^{76}$, C.L. Lampen 6 , W. Lampl ${ }^{6}$, E. Lancon ${ }^{135}$, U. Landgraf ${ }^{47}$, M.P.J. Landon ${ }^{74}$, J.L. Lane ${ }^{81}$, V.S. Lang ${ }^{57 a}$, C. Lange ${ }^{41}$, A.J. Lankford ${ }^{162}$, F. Lanni ${ }^{24}$, K. Lantzsch ${ }^{174}$, S. Laplace ${ }^{77}$, C. Lapoire ${ }^{20}$, J.F. Laporte ${ }^{135}$, T. Lari ${ }^{88 a}$, A. Larner ${ }^{117}$, M. Lassnig ${ }^{29}$, P. Laurelli ${ }^{46}$, V. Lavorini ${ }^{36 a, 36 b}$, W. Lavrijsen ${ }^{14}$, P. Laycock ${ }^{72}$, O. Le Dortz ${ }^{77}$, E. Le Guirriec ${ }^{82}$, C. Le Maner ${ }^{157}$, E. Le Menedeu ${ }^{11}$, T. LeCompte ${ }^{5}$, F. Ledroit-Guillon ${ }^{54}$, H. Lee ${ }^{104}$, J.S.H. Lee ${ }^{115}$, S.C. Lee $^{150}$, L. Lee ${ }^{175}$, M. Lefebvre ${ }^{168}$, M. Legendre ${ }^{135}$, F. Legger ${ }^{97}$, C. Leggett ${ }^{14}$, M. Lehmacher ${ }^{20}$, G. Lehmann Miotto ${ }^{29}$, X. Lei ${ }^{6}$, M.A.L. Leite ${ }^{23 d}$, R. Leitner ${ }^{125}$, D. Lellouch ${ }^{171}$, B. Lemmer ${ }^{53}$, V. Lendermann ${ }^{57 a}$, K.J.C. Leney ${ }^{144 b}$, T. Lenz ${ }^{104}$, G. Lenzen ${ }^{174}$, B. Lenzi ${ }^{29}$, K. Leonhardt ${ }^{43}$, S. Leontsinis ${ }^{9}$, F. Lepold ${ }^{57 a}$, C. Leroy ${ }^{92}$, J-R. Lessard ${ }^{168}$, C.G. Lester ${ }^{27}$, C.M. Lester ${ }^{119}$, J. Levêque ${ }^{4}$, D. Levin ${ }^{86}$, L.J. Levinson ${ }^{171}$, A. Lewis ${ }^{117}$, G.H. Lewis ${ }^{107}$, A.M. Leyko ${ }^{20}$, M. Leyton ${ }^{15}$, B. Li ${ }^{82}$,

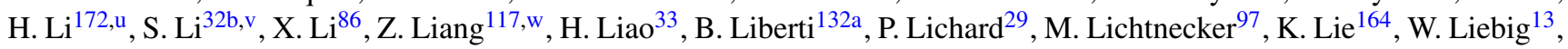
C. Limbach $^{20}$, A. Limosani ${ }^{85}$, M. Limper ${ }^{61}$, S.C. Lin $^{150, x}$, F. Linde ${ }^{104}$, J.T. Linnemann ${ }^{87}$, E. Lipeles ${ }^{119}$, A. Lipniacka ${ }^{13}$,

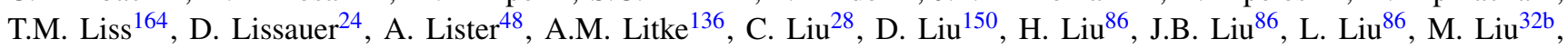
Y. Liu ${ }^{32 b}$, M. Livan ${ }^{118 a, 118 b}$, S.S.A. Livermore ${ }^{117}$, A. Lleres ${ }^{54}$, J. Llorente Merino ${ }^{79}$, S.L. Lloyd ${ }^{74}$, E. Lobodzinska ${ }^{41}$, P. Loch $^{6}$, W.S. Lockman ${ }^{136}$, T. Loddenkoetter ${ }^{20}$, F.K. Loebinger ${ }^{81}$, A. Loginov ${ }^{175}$, C.W. Loh ${ }^{167}$, T. Lohse ${ }^{15}$, K. Lohwasser ${ }^{47}$, M. Lokajicek ${ }^{124}$, V.P. Lombardo ${ }^{4}$, R.E. Long ${ }^{70}$, L. Lopes ${ }^{123 a}$, D. Lopez Mateos ${ }^{56}$, J. Lorenz ${ }^{97}$, N. Lorenzo Martinez ${ }^{114}$, M. Losada ${ }^{161}$, P. Loscutoff ${ }^{14}$, F. Lo Sterzo ${ }^{131 a, 131 b}$, M.J. Losty ${ }^{158 a}$, X. Lou ${ }^{40}$, A. Lounis ${ }^{114}$, K.F. Loureiro ${ }^{161}$, J. Love ${ }^{21}$, P.A. Love $^{70}$, A.J. Lowe ${ }^{142, e}$, F. Lu ${ }^{32 a}$, H.J. Lubatti137, C. Luci ${ }^{131 a, 131 b}$, A. Lucotte ${ }^{54}$, A. Ludwig ${ }^{43}$, D. Ludwig ${ }^{41}$, I. Ludwig $^{47}$, J. Ludwig ${ }^{47}$, F. Luehring ${ }^{59}$, G. Luijckx ${ }^{104}$, W. Lukas ${ }^{60}$, D. Lumb ${ }^{47}$, L. Luminari ${ }^{131 a}$, E. Lund ${ }^{116}$, B. Lund-Jensen ${ }^{146}$, B. Lundberg ${ }^{78}$, J. Lundberg ${ }^{145 a, 145 b}$, O. Lundberg ${ }^{145 a, 145 b}$, J. Lundquist ${ }^{35}$, M. Lungwitz ${ }^{80}$, D. Lynn ${ }^{24}$, E. Lytken ${ }^{78}$, H. Ma $^{24}$, L.L. $\mathrm{Ma}^{172}$, G. Maccarrone ${ }^{46}$, A. Macchiolo ${ }^{98}$, B. Maček ${ }^{73}$, J. Machado Miguens ${ }^{123 a}$, R. Mackeprang ${ }^{35}$, R.J. Madaras ${ }^{14}$, H.J. Maddocks ${ }^{70}$, W.F. Mader ${ }^{43}$, R. Maenner5 ${ }^{57 \mathrm{c}}$, T. Maeno ${ }^{24}$, P. Mättig ${ }^{174}$, S. Mättig ${ }^{41}$, L. Magnoni ${ }^{29}$, E. Magradze ${ }^{53}$, K. Mahboubi ${ }^{47}$, S. Mahmoud ${ }^{72}$, G. Mahout ${ }^{17}$, C. Maiani ${ }^{135}$, C. Maidantchik ${ }^{23 a}$, A. Maio ${ }^{123 a, b}$, S. Majewski ${ }^{24}$, Y. Makida ${ }^{64}$, N. Makovec ${ }^{114}$, P. Mal ${ }^{135}$, B. Malaescu ${ }^{29}$, Pa. Malecki ${ }^{38}$, P. Malecki ${ }^{38}$, V.P. Maleev ${ }^{120}$, F. Malek ${ }^{54}$, U. Mallik ${ }^{61}$, D. Malon ${ }^{5}$, C. Malone ${ }^{142}$, S. Maltezos ${ }^{9}$, V. Malyshev ${ }^{106}$, S. Malyukov ${ }^{29}$, R. Mameghani ${ }^{97}$, J. Mamuzic ${ }^{12 b}$, A. Manabe ${ }^{64}$, L. Mandelli $^{88 a}$, I. Mandić ${ }^{73}$, R. Mandrysch ${ }^{15}$, J. Maneira ${ }^{123 a}$, P.S. Mangeard ${ }^{87}$, L. Manhaes de Andrade Filho ${ }^{23 b}$, J.A. Manjarres Ramos ${ }^{135}$, A. Mann ${ }^{53}$, P.M. Manning ${ }^{136}$, A. Manousakis-Katsikakis ${ }^{8}$, B. Mansoulie ${ }^{135}$, A. Mapelli ${ }^{29}$, L. Mapelli ${ }^{29}$, 
${\text { L. } \operatorname{March}^{79} \text {, J.F. Marchand }}^{28}$, F. Marchese ${ }^{132 a, 132 b}$, G. Marchiori ${ }^{77}$, M. Marcisovsky ${ }^{124}$, C.P. Marino ${ }^{168}$, F. Marroquim ${ }^{23 a}$, Z. Marshall ${ }^{29}$, F.K. Martens ${ }^{157}$, L.F. Marti ${ }^{16}$, S. Marti-Garcia ${ }^{166}$, B. Martin ${ }^{29}$, B. Martin ${ }^{87}$, J.P. Martin ${ }^{92}$, T.A. Martin ${ }^{17}$, V.J. Martin ${ }^{45}$, B. Martin dit Latour ${ }^{48}$, S. Martin-Haugh ${ }^{148}$, M. Martinez ${ }^{11}$, V. Martinez Outschoorn ${ }^{56}$, A.C. Martyniuk ${ }^{168}$, M. Marx ${ }^{81}$, F. Marzano ${ }^{131 a}$, A. Marzin ${ }^{110}$, L. Masetti8 ${ }^{80}$, T. Mashimo ${ }^{154}$, R. Mashinistov ${ }^{93}$, J. Masik ${ }^{81}$, A.L. Maslennikov ${ }^{106}$, I. Massa $^{19 a, 19 b}$, G. Massaro ${ }^{104}$, N. Massol ${ }^{4}$, P. Mastrandrea ${ }^{147}$, A. Mastroberardino ${ }^{36 a, 36 b}$, T. Masubuchi ${ }^{154}$, P. Matricon ${ }^{114}$, H. Matsunaga ${ }^{154}$, T. Matsushita ${ }^{65}$, C. Mattravers ${ }^{117, \mathrm{c}}$, J. Maurer ${ }^{82}$, S.J. Maxfield ${ }^{72}$, A. Mayne ${ }^{138}$, R. Mazini ${ }^{150}$, M. Mazur ${ }^{20}$, L. Mazzaferro ${ }^{132 a, 132 b}$, M. Mazzanti ${ }^{88 a}$, S.P. Mc Kee ${ }^{86}$, A. McCarn ${ }^{164}$, R.L. McCarthy ${ }^{147}$, T.G. McCarthy ${ }^{28}$, N.A. McCubbin $^{128}$, K.W. McFarlane ${ }^{55, *}$, J.A. Mcfayden ${ }^{138}$, G. Mchedlidze ${ }^{50 b}$, T. Mclaughlan ${ }^{17}$, S.J. McMahon ${ }^{128}$, R.A. McPherson $^{168, k}$, A. Meade ${ }^{83}$, J. Mechnich ${ }^{104}$, M. Mechtel ${ }^{174}$, M. Medinnis ${ }^{41}$, R. Meera-Lebbai ${ }^{110}$, T. Meguro ${ }^{115}$, R. Mehdiyev ${ }^{92}$, S. Mehlhase ${ }^{35}$, A. Mehta ${ }^{72}$, K. Meier ${ }^{57 a}$, B. Meirose ${ }^{78}$, C. Melachrinos ${ }^{30}$, B.R. Mellado Garcia ${ }^{172}$, F. Meloni ${ }^{88 a, 88 b}$, L. Mendoza Navas ${ }^{161}$, Z. Meng ${ }^{150, u}$, A. Mengarelli ${ }^{19 a}$,19b, S. Menke ${ }^{98}$, E. Meoni ${ }^{160}$, K.M. Mercurio ${ }^{56}$, P. Mermod ${ }^{48}$, L. Merola ${ }^{101 a, 101 b}$, C. Meroni ${ }^{88 a}$, F.S. Merritt ${ }^{30}$, H. Merritt ${ }^{108}$, A. Messina ${ }^{29, y}$, J. Metcalfe $^{102}$, A.S. Mete ${ }^{162}$, C. Meyer ${ }^{80}$, C. $M_{e y e r}{ }^{30}$, J-P. Meyer ${ }^{135}$, J. Meyer ${ }^{173}$, J. Meyer ${ }^{53}$, T.C. Meyer $^{29}$, J. Miao ${ }^{32 \mathrm{~d}}$, S. Michal ${ }^{29}$, L. Micu ${ }^{25 a}$, R.P. Middleton ${ }^{128}$, S. Migas ${ }^{72}$, L. Mijović ${ }^{135}$, G. Mikenberg ${ }^{171}$, M. Mikestikova ${ }^{124}$, M. Mikuž ${ }^{73}$, D.W. Miller ${ }^{30}$, R.J. Miller ${ }^{87}$, W.J. Mills ${ }^{167}$, C. Mills ${ }^{56}$, A. Milov ${ }^{171}$, D.A. Milstead ${ }^{145 a, 145 b}$, D. Milstein ${ }^{171}$, A.A. Minaenko ${ }^{127}$, M. Miñano Moya ${ }^{166}$, I.A. Minashvili ${ }^{63}$, A.I. Mincer ${ }^{107}$, B. Mindur ${ }^{37}$, M. Mineev ${ }^{63}$, Y. Ming $^{172}$, L.M. Mir ${ }^{11}$, G. Mirabelli131a ${ }^{131}$ J. Mitrevski ${ }^{136}$, V.A. Mitsou ${ }^{166}$, S. Mitsui ${ }^{64}$, P.S. Miyagawa ${ }^{138}$, J.U. Mjörnmark ${ }^{78}$, T. Moa ${ }^{145 a, 145 b}$, V. Moeller ${ }^{27}$, K. Mönig ${ }^{41}$, N. Möser ${ }^{20}$, S. Mohapatra ${ }^{147}$, W. Mohr ${ }^{47}$, R. Moles-Valls ${ }^{166}$, A. Molfetas ${ }^{29}$, J. Monk ${ }^{76}$, E. Monnier ${ }^{82}$, J. Montejo Berlingen ${ }^{11}$, F. Monticelli ${ }^{69}$, S. Monzani $^{19 a, 19 b}$, R.W. Moore ${ }^{2}$, G.F. Moorhead ${ }^{85}$, C. Mora Herrera ${ }^{48}$, A. Moraes ${ }^{52}$, N. Morange ${ }^{135}$, J. Morel ${ }^{53}$, G. Morello ${ }^{36 a, 36 b}$, D. Moreno ${ }^{80}$, M. Moreno Llácer ${ }^{166}$, P. Morettini ${ }^{49 a}$, M. Morgenstern ${ }^{43}$, M. Morii ${ }^{56}$, A.K. Morley ${ }^{29}$, G. Mornacchi ${ }^{29}$, J.D. Morris ${ }^{74}$, L. Morvaj ${ }^{100}$, H.G. Moser ${ }^{98}$, M. Mosidze ${ }^{50 b}$, J. Moss ${ }^{108}$, R. Mount ${ }^{142}$, E. Mountricha ${ }^{9, z}$, S.V. Mouraviev ${ }^{93, *}$, E.J.W. Moyse ${ }^{83}$, F. Mueller ${ }^{57 a}$, J. Mueller ${ }^{122}$, K. Mueller ${ }^{20}$, T.A. Müller ${ }^{97}$, T. Mueller ${ }^{80}$, D. Muenstermann ${ }^{29}$, Y. Munwes ${ }^{152}$, W.J. Murray ${ }^{128}$, I. Mussche ${ }^{104}$, E. Musto ${ }^{101 a, 101 b}$, A.G. Myagkov ${ }^{127}$, M. Myska ${ }^{124}$, J. Nadal ${ }^{11}$, K. Nagai ${ }^{159}$, R. Nagai $^{156}$, K. Nagano ${ }^{64}$, A. Nagarkar ${ }^{108}$, Y. Nagasaka ${ }^{58}$, M. Nagel ${ }^{98}$, A.M. Nairz ${ }^{29}$, Y. Nakahama ${ }^{29}$, K. Nakamura ${ }^{154}$, T. Nakamura ${ }^{154}$, I. Nakano ${ }^{109}$, G. Nanava ${ }^{20}$, A. Napier ${ }^{160}$, R. Narayan ${ }^{57 b}$, M. Nash ${ }^{76, c}$, T. Nattermann ${ }^{20}$, T. Naumann ${ }^{41}$, G. Navarro ${ }^{161}$, H.A. Neal ${ }^{86}$, P.Yu. Nechaeva ${ }^{93}$, T.J. Neep ${ }^{81}$, A. Negri ${ }^{118 a}{ }^{118 b}$, G. Negri ${ }^{29}$, M. Negrini ${ }^{19 a}$, S. Nektarijevic ${ }^{48}$, A. Nelson ${ }^{162}$, T.K. Nelson ${ }^{142}$, S. Nemecek ${ }^{124}$, P. Nemethy ${ }^{107}$, A.A. Nepomuceno ${ }^{23 a}$, M. Nessi ${ }^{29, a a}$, M.S. Neubauer ${ }^{164}$, A. Neusiedl ${ }^{80}$, R.M. Neves ${ }^{107}$, P. Nevski ${ }^{24}$, F.M. Newcomer ${ }^{119}$, P.R. Newman ${ }^{17}$, V. Nguyen Thi Hong ${ }^{135}$, R.B. Nickerson $^{117}$, R. Nicolaidou ${ }^{135}$, B. Nicquevert ${ }^{29}$, F. Niedercorn ${ }^{114}$, J. Nielsen ${ }^{136}$, N. Nikiforou ${ }^{34}$, A. Nikiforov ${ }^{15}$, V. Nikolaenko $^{127}$, I. Nikolic-Audit ${ }^{77}$, K. Nikolics ${ }^{48}$, K. Nikolopoulos ${ }^{17}$, H. Nilsen $^{47}$, P. Nilsson ${ }^{7}$, Y. Ninomiya ${ }^{154}$, A. Nisati ${ }^{131 a}$, R. Nisius ${ }^{98}$, T. Nobe ${ }^{156}$, L. Nodulman ${ }^{5}$, M. Nomachi ${ }^{115}$, I. Nomidis ${ }^{153}$, S. Norberg ${ }^{110}$, M. Nordberg ${ }^{29}$, P.R. Norton ${ }^{128}$, J. Novakova $^{125}$, M. Nozaki ${ }^{64}$, L. Nozka ${ }^{112}$, I.M. Nugent ${ }^{158 a}$, A.-E. Nuncio-Quiroz ${ }^{20}$, G. Nunes Hanninger ${ }^{85}$, T. Nunnemann ${ }^{97}$, ${\text { E. } \text { Nurse }^{76} \text {, B.J. O’Brien }}^{45}$, S.W. O’Neale ${ }^{17, *}$, D.C. O’Neil ${ }^{141}$, V. O'Shea ${ }^{52}$, L.B. Oakes ${ }^{97}$, F.G. Oakham ${ }^{28, d}$, H. Oberlack ${ }^{98}$, J. Ocariz ${ }^{77}$, A. Ochi ${ }^{65}$, S. Oda ${ }^{68}$, S. Odaka ${ }^{64}$, J. Odier ${ }^{82}$, H. Ogren ${ }^{59}$, A. $\mathrm{Oh}^{81}$, S.H. Oh ${ }^{44}$, C.C. Ohm ${ }^{29}$, T. Ohshima ${ }^{100}$, H. Okawa ${ }^{24}$, Y. Okumura $^{30}$, T. Okuyama ${ }^{154}$, A. Olariu ${ }^{25 a}$, A.G. Olchevski ${ }^{63}$, S.A. Olivares Pino ${ }^{31 a}$, M. Oliveira ${ }^{123 a, h}$, D. Oliveira Damazio ${ }^{24}$, E. Oliver Garcia ${ }^{166}$, D. Olivito ${ }^{119}$, A. Olszewski ${ }^{38}$, J. Olszowska ${ }^{38}$, A. Onofre ${ }^{123 a, a b}$, P.U.E. Onyisi $^{30}$, C.J. Oram ${ }^{158 a}$, M.J. Oreglia ${ }^{30}$, Y. Oren ${ }^{152}$, D. Orestano ${ }^{133 a, 133 b}$, N. Orlando ${ }^{71 a, 71 b}$, I. Orlov ${ }^{106}$, C. Oropeza Barrera ${ }^{52}$,

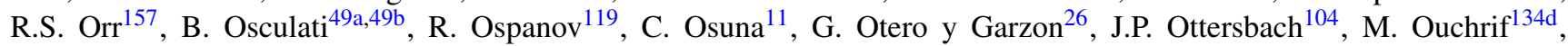
E.A. Ouellette ${ }^{168}$, F. Ould-Saada ${ }^{116}$, A. Ouraou ${ }^{135}$, Q. Ouyang ${ }^{32 a}$, A. Ovcharova ${ }^{14}$, M. Owen ${ }^{81}$, S. Owen ${ }^{138}$, V.E. Ozcan $^{18 a}$, N. Ozturk ${ }^{7}$, A. Pacheco Pages ${ }^{11}$, C. Padilla Aranda ${ }^{11}$, S. Pagan Griso ${ }^{14}$, E. Paganis ${ }^{138}$, C. Pahl ${ }^{98}$, F. Paige ${ }^{24}$, P. Pais $^{83}$, K. Pajchel ${ }^{116}$, G. Palacino ${ }^{158 b}$, C.P. Paleari ${ }^{6}$, S. Palestini ${ }^{29}$, D. Pallin ${ }^{33}$, A. Palma ${ }^{123 a}$, J.D. Palmer ${ }^{17}$, Y.B. Pan ${ }^{172}$, E. Panagiotopoulou ${ }^{9}$, P. Pani ${ }^{104}$, N. Panikashvili ${ }^{86}$, S. Panitkin ${ }^{24}$, D. Pantea ${ }^{25 a}$, A. Papadelis ${ }^{145 a}$, Th.D. Papadopoulou ${ }^{9}$, A. Paramonov ${ }^{5}$, D. Paredes Hernandez ${ }^{33}$, W. Park ${ }^{24, a c}$, M.A. Parker ${ }^{27}$, F. Parodi ${ }^{49 a, 49 b}$, J.A. Parsons ${ }^{34}$, U. Parzefall ${ }^{47}$, S. Pashapour ${ }^{53}$, E. Pasqualucci131a, S. Passaggio ${ }^{49 a}$, A. Passeri ${ }^{133 a}$, F. Pastore ${ }^{133 a, 133 b, *}$, Fr. Pastore ${ }^{75}$, G. Pásztor ${ }^{48, a d}$, S. Pataraia ${ }^{174}$, N. Patel $^{149}$, J.R. Pater ${ }^{81}$, S. Patricelli ${ }^{101 a, 101 b}$, T. Pauly ${ }^{29}$, M. Pecsy ${ }^{143 a}$, M.I. Pedraza Morales ${ }^{172}$, S.V. Pele-

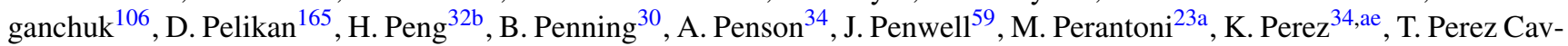
alcanti $^{41}$, E. Perez Codina ${ }^{158 a}$, M.T. Pérez García-Estañ ${ }^{166}$, V. Perez Reale ${ }^{34}$, L. Perini ${ }^{88 a}{ }^{88 b}$, H. Pernegger ${ }^{29}$, R. Perrino ${ }^{71 a}$, P. Perrodo ${ }^{4}$, V.D. Peshekhonov ${ }^{63}$, K. Peters ${ }^{29}$, B.A. Petersen ${ }^{29}$, J. Petersen ${ }^{29}$, T.C. Petersen ${ }^{35}$, E. Petit ${ }^{4}$, A. Petridis ${ }^{153}$, C. Petridou ${ }^{153}$, E. Petrolo ${ }^{131 a}$, F. Petrucci ${ }^{133 a, 133 b}$, D. Petschull ${ }^{41}$, M. Petteni ${ }^{141}$, R. Pezoa ${ }^{31 b}$, A. Phan ${ }^{85}$, P.W. Phillips ${ }^{128}$,

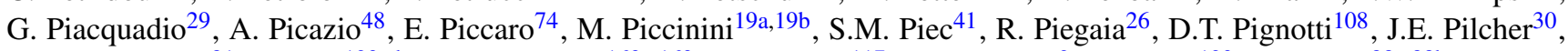
A.D. Pilkington ${ }^{81}$, J. Pina ${ }^{123 a, b}$, M. Pinamonti ${ }^{163 a, 163 c}$, A. Pinder ${ }^{117}$, J.L. Pinfold ${ }^{2}$, B. Pinto ${ }^{123 a}$, C. Pizio ${ }^{88 a, 88 b}$, M. Plamondon $^{168}$, M.-A. Pleier ${ }^{24}$, E. Plotnikova ${ }^{63}$, A. Poblaguev ${ }^{24}$, S. Poddar ${ }^{57 a}$, F. Podlyski ${ }^{33}$, L. Poggioli ${ }^{114}$, M. Pohl ${ }^{48}$, G. Polesello ${ }^{118 a}$, A. Policicchio ${ }^{36 a, 36 b}$, A. Polini ${ }^{19 a}$, J. Poll ${ }^{74}$, V. Polychronakos ${ }^{24}$, D. Pomeroy ${ }^{22}$, K. Pommès ${ }^{29}$, L. Pontecorvo $^{131 a}$, B.G. Pope ${ }^{87}$, G.A. Popeneciu ${ }^{25 a}$, D.S. Popovic ${ }^{12 a}$, A. Poppleton ${ }^{29}$, X. Portell Bueso ${ }^{29}$, G.E. Pospelov ${ }^{98}$, 
S. Pospisil ${ }^{126}$, I.N. Potrap ${ }^{98}$, C.J. Potter ${ }^{148}$, C.T. Potter ${ }^{113}$, G. Poulard ${ }^{29}$, J. Poveda ${ }^{59}$, V. Pozdnyakov ${ }^{63}$, R. Prabhu ${ }^{76}$, P. Pralavorio ${ }^{82}$, A. Pranko ${ }^{14}$, S. Prasad ${ }^{29}$, R. Pravahan ${ }^{24}$, S. Prell ${ }^{62}$, K. Pretzl ${ }^{16}$, D. Price ${ }^{59}$, J. Price ${ }^{72}$, L.E. Price ${ }^{5}$, D. Prieur ${ }^{122}$, M. Primavera ${ }^{71 a}$, K. Prokofiev ${ }^{107}$, F. Prokoshin ${ }^{31 b}$, S. Protopopescu ${ }^{24}$, J. Proudfoot ${ }^{5}$, X. Prudent ${ }^{43}$, M. Przybycien $^{37}$, H. Przysiezniak ${ }^{4}$, S. Psoroulas ${ }^{20}$, E. Ptacek ${ }^{113}$, E. Pueschel ${ }^{83}$, J. Purdham ${ }^{86}$, M. Purohit ${ }^{24, a c}$, P. Puzo $^{114}$, Y. Pylypchenko ${ }^{61}$, J. Qian ${ }^{86}$, A. Quadt ${ }^{53}$, D.R. Quarrie ${ }^{14}$, W.B. Quayle ${ }^{172}$, F. Quinonez ${ }^{31 a}$, M. Raas ${ }^{103}$, V. Radeka ${ }^{24}$, V. Radescu ${ }^{41}$, P. Radloff ${ }^{113}$, T. Rador ${ }^{18 a}$, F. Ragusa ${ }^{88 a, 88 b}$, G. Rahal ${ }^{177}$, A.M. Rahimi ${ }^{108}$, D. Rahm ${ }^{24}$, S. Rajagopalan ${ }^{24}$, M. Rammensee ${ }^{47}$, M. Rammes ${ }^{140}$, A.S. Randle-Conde ${ }^{39}$, K. Randrianarivony ${ }^{28}$, F. Rauscher ${ }^{97}$, T.C. Rave ${ }^{47}$, M. Raymond ${ }^{29}$, A.L. Read ${ }^{116}$, D.M. Rebuzzi ${ }^{18 a, 118 b}$, A. Redelbach ${ }^{173}$, G. Redlinger ${ }^{24}$, R. Reece ${ }^{119}$, K. Reeves ${ }^{40}$, E. Reinherz-Aronis ${ }^{152}$, A. Reinsch ${ }^{113}$, I. Reisinger ${ }^{42}$, C. Rembser $^{29}$, Z.L. Ren ${ }^{150}$, A. Renaud ${ }^{114}$, M. Rescigno ${ }^{131 a}$, S. Resconi ${ }^{88 a}$, B. Resende ${ }^{135}$, P. Reznicek ${ }^{97}$, R. Rezvani ${ }^{157}$, R. Richter ${ }^{98}$, E. Richter-Was ${ }^{4, a f}$, M. Ridel ${ }^{77}$, M. Rijpstra ${ }^{104}$, M. Rijssenbeek ${ }^{147}$, A. Rimoldi ${ }^{118 a, 118 b}$, L. Rinaldi ${ }^{19 a}$, R.R. Rios ${ }^{39}$, I. Riu ${ }^{11}$, G. Rivoltella ${ }^{88 a, 88 b}$, F. Rizatdinova ${ }^{111}$, E. Rizvi ${ }^{74}$, S.H. Robertson ${ }^{84, k}$, A. RobichaudVeronneau $^{117}$, D. Robinson ${ }^{27}$, J.E.M. Robinson ${ }^{81}$, A. Robson ${ }^{52}$, J.G. Rocha de Lima ${ }^{105}$, C. Roda ${ }^{121 a, 121 b}$, D. Roda Dos San$\operatorname{tos}^{29}$, A. Roe ${ }^{53}$, S. Roe ${ }^{29}$, O. Røhne ${ }^{116}$, S. Rolli ${ }^{160}$, A. Romaniouk ${ }^{95}$, M. Romano ${ }^{19 a, 19 b}$, G. Romeo ${ }^{26}$, E. Romero Adam $^{166}$, L. Roos ${ }^{77}$, E. Ros ${ }^{166}$, S. Rosati ${ }^{131 a}$, K. Rosbach ${ }^{48}$, A. Rose ${ }^{148}$, M. Rose ${ }^{75}$, G.A. Rosenbaum ${ }^{157}$, E.I. Rosenberg $^{62}$, P.L. Rosendahl ${ }^{13}$, O. Rosenthal ${ }^{140}$, L. Rosselet ${ }^{48}$, V. Rossetti ${ }^{11}$, E. Rossi ${ }^{131 a, 131 b}$, L.P. Rossi ${ }^{49 a}$, M. Rotaru ${ }^{25 a}$, I. Roth ${ }^{171}$, J. Rothberg ${ }^{137}$, D. Rousseau ${ }^{114}$, C.R. Royon ${ }^{135}$, A. Rozanov ${ }^{82}$, Y. Rozen ${ }^{151}$, X. Ruan ${ }^{32 a, a g}$, F. Rubbo ${ }^{11}$, I. Rubinskiy $^{41}$, B. Ruckert ${ }^{97}$, N. Ruckstuhl ${ }^{104}$, V.I. Rud ${ }^{96}$, C. Rudolph ${ }^{43}$, G. Rudolph ${ }^{60}$, F. Rühr ${ }^{6}$, A. Ruiz-Martinez ${ }^{62}$, L. Rumyant$\operatorname{sev}^{63}$, Z. Rurikova ${ }^{47}$, N.A. Rusakovich ${ }^{63}$, J.P. Rutherfoord ${ }^{6}$, C. Ruwiedel ${ }^{14, *}$, P. Ruzicka ${ }^{124}$, Y.F. Ryabov ${ }^{120}$, P. Ryan ${ }^{87}$, M. Rybar ${ }^{125}$, G. Rybkin ${ }^{114}$, N.C. Ryder ${ }^{117}$, A.F. Saavedra ${ }^{149}$, I. Sadeh ${ }^{152}$, H.F-W. Sadrozinski ${ }^{136}$, R. Sadykov ${ }^{63}$, F. Safai Tehrani ${ }^{131 a}$, H. Sakamoto ${ }^{154}$, G. Salamanna ${ }^{74}$, A. Salamon ${ }^{132 a}$, M. Saleem ${ }^{110}$, D. Salek ${ }^{29}$, D. Salihagic ${ }^{98}$, A. Salnikov ${ }^{142}$, J. Salt ${ }^{166}$, B.M. Salvachua Ferrando ${ }^{5}$, D. Salvatore ${ }^{36 a, 36 b}$, F. Salvatore ${ }^{148}$, A. Salvucci ${ }^{103}$, A. Salzburger ${ }^{29}$, D. Sampsonidis $^{153}$, B.H. Samset ${ }^{116}$, A. Sanchez ${ }^{101 a, 101 b}$, V. Sanchez Martinez ${ }^{166}$, H. Sandaker ${ }^{13}$, H.G. Sander ${ }^{80}$, M.P. Sanders ${ }^{97}$, M. Sandhoff ${ }^{174}$, T. Sandoval ${ }^{27}$, C. Sandoval ${ }^{161}$, R. Sandstroem ${ }^{98}$, D.P.C. Sankey ${ }^{128}$, A. Sansoni ${ }^{46}$, C. Santamarina Rios ${ }^{84}$, C. Santoni ${ }^{33}$, R. Santonico ${ }^{132 a, 132 b}$, H. Santos ${ }^{123 a}$, J.G. Saraiva ${ }^{123 a}$, T. Sarangi ${ }^{172}$, E. Sarkisyan-Grinbaum ${ }^{7}$, F. Sarri ${ }^{121 a, 121 b}$, G. Sartisohn ${ }^{174}$, O. Sasaki ${ }^{64}$, Y. Sasaki ${ }^{154}$, N. Sasao ${ }^{66}$, I. Satsounkevitch ${ }^{89}$, G. Sauvage, ${ }^{4 *}$, E. Sauvan ${ }^{4}$, J.B. Sauvan ${ }^{114}$, P. Savard ${ }^{157, d}$, V. Savinov ${ }^{122}$, D.O. Savu ${ }^{29}$, L. Sawyer ${ }^{24, m}$, D.H. Saxon ${ }^{52}$, J. Saxon ${ }^{119}$, C. Sbarra ${ }^{19 a}$, A. Sbrizzi ${ }^{19 a, 19 b}$, D.A. Scannicchio ${ }^{162}$, M. Scarcella ${ }^{149}$, J. Schaarschmidt ${ }^{114}$, P. Schacht ${ }^{98}$, D. Schaefer ${ }^{119}$, U. Schäfer ${ }^{80}$, S. Schaepe ${ }^{20}$, S. Schaetzel ${ }^{57 b}$, A.C. Schaffer ${ }^{114}$, D. Schaile ${ }^{97}$, R.D. Schamberger ${ }^{147}$, A.G. Schamov ${ }^{106}$, V. Scharf ${ }^{57 a}$, V.A. Schegelsky $^{120}$, D. Scheirich ${ }^{86}$, M. Schernau ${ }^{162}$, M.I. Scherzer ${ }^{34}$, C. Schiavi ${ }^{49 a, 49 b}$, J. Schieck ${ }^{97}$, M. Schioppa ${ }^{36 a, 36 b}$, S. Schlenker ${ }^{29}$, E. Schmidt ${ }^{47}$, K. Schmieden ${ }^{20}$, C. Schmitt ${ }^{80}$, S. Schmitt ${ }^{57 b}$, M. Schmitz ${ }^{20}$, B. Schneider ${ }^{16}$, U. Schnoor ${ }^{43}$, A. Schoening ${ }^{57 b}$, A.L.S. Schorlemmer ${ }^{53}$, M. Schott ${ }^{29}$, D. Schouten ${ }^{158 a}$, J. Schovancova ${ }^{124}$, M. Schram ${ }^{84}$, C. Schroeder ${ }^{80}$, N. Schroer ${ }^{57 c}$, M.J. Schultens ${ }^{20}$, J. Schultes ${ }^{174}$, H.-C. Schultz-Coulon ${ }^{57 a}$, H. Schulz ${ }^{15}$, M. Schumacher ${ }^{47}$, B.A. Schumm ${ }^{136}$, Ph. Schune $^{135}$, C. Schwanenberger ${ }^{81}$, A. Schwartzman ${ }^{142}$, Ph. Schwemling ${ }^{77}$, R. Schwienhorst ${ }^{87}$, R. Schwierz ${ }^{43}$, J. Schwindling ${ }^{135}$, T. Schwindt ${ }^{20}$, M. Schwoerer ${ }^{4}$, G. Sciolla ${ }^{22}$, W.G. Scott ${ }^{128}$, J. Searcy ${ }^{113}$, G. Sedov ${ }^{41}$, E. Sedykh ${ }^{120}$, S.C. Seidel ${ }^{102}$, A. Sei$\operatorname{den}^{136}$, F. Seifert ${ }^{43}$, J.M. Seixas ${ }^{23 a}$, G. Sekhniaidze ${ }^{101 a}$, S.J. Sekula ${ }^{39}$, K.E. Selbach ${ }^{45}$, D.M. Seliverstov ${ }^{120}$, B. Sellden ${ }^{145 a}$, G. Sellers ${ }^{72}$, M. Seman ${ }^{143 b}$, N. Semprini-Cesari ${ }^{19 a, 19 b}$, C. Serfon ${ }^{97}$, L. Serin ${ }^{114}$, L. Serkin ${ }^{53}$, R. Seuster ${ }^{98}$, H. Severini ${ }^{110}$, A. Sfyrla ${ }^{29}$, E. Shabalina ${ }^{53}$, M. Shamim ${ }^{113}$, L.Y. Shan ${ }^{32 a}$, J.T. Shank ${ }^{21}$, Q.T. Shao ${ }^{85}$, M. Shapiro ${ }^{14}$, P.B. Shatalov ${ }^{94}$, K. Shaw ${ }^{163 a, 163 c}$, D. Sherman ${ }^{175}$, P. Sherwood ${ }^{76}$, A. Shibata ${ }^{107}$, S. Shimizu ${ }^{29}$, M. Shimojima ${ }^{99}$, T. Shin ${ }^{55}$, M. Shiyakova ${ }^{63}$, A. Shmeleva ${ }^{93}$, M.J. Shochet ${ }^{30}$, D. Short ${ }^{117}$, S. Shrestha ${ }^{62}$, E. Shulga ${ }^{95}$, M.A. Shupe ${ }^{6}$, P. Sicho ${ }^{124}$, A. Sidoti ${ }^{131 a}$, F. Siegert ${ }^{47}$, Dj. Sijacki ${ }^{12 a}$, O. Silbert ${ }^{171}$, J. Silva ${ }^{123 a}$, Y. Silver ${ }^{152}$, D. Silverstein ${ }^{142}$, S.B. Silverstein ${ }^{145 a}$, V. Simak ${ }^{126}$, O. Simard ${ }^{135}$, Lj. Simic ${ }^{12 a}$, S. Simion ${ }^{114}$, E. Simioni ${ }^{80}$, B. Simmons ${ }^{76}$, R. Simoniello ${ }^{88 a, 88 b}$, M. Simonyan ${ }^{35}$, P. Sinervo ${ }^{157}$, N.B. Sinev ${ }^{113}$, V. Sipica ${ }^{140}$, G. Siragusa ${ }^{173}$, A. Sircar ${ }^{24}$, A.N. Sisakyan ${ }^{63,{ }^{*}}$, S.Yu. Sivoklokov ${ }^{96}$, J. Sjölin ${ }^{145 a, 145 b}$, T.B. Sjursen ${ }^{13}$, L.A. Skinnari $^{14}$, H.P. Skottowe ${ }^{56}$, K. Skovpen ${ }^{106}$, P. Skubic ${ }^{110}$, M. Slater ${ }^{17}$, T. Slavicek ${ }^{126}$, K. Sliwa ${ }^{160}$, V. Smakhtin ${ }^{171}$, B.H. Smart ${ }^{45}$, S.Yu. Smirnov ${ }^{95}$, Y. Smirnov ${ }^{95}$, L.N. Smirnova ${ }^{96}$, O. Smirnova $^{78}$, B.C. Smith $^{56}$, D. Smith ${ }^{142}$, K.M. Smith ${ }^{52}$, M. Smizanska $^{70}$, K. Smolek ${ }^{126}$, A.A. Snesarev ${ }^{93}$, S.W. Snow ${ }^{81}$, J. Snow ${ }^{110}$, S. Snyder ${ }^{24}$, R. Sobie ${ }^{168, k}$, J. Sodomka ${ }^{126}$, A. Soffer ${ }^{152}$, C.A. Solans ${ }^{166}$, M. Solar ${ }^{126}$, J. Solc ${ }^{126}$, E.Yu. Soldatov ${ }^{95}$, U. Soldevila ${ }^{166}$, E. Solfaroli Camillocci131a,131b , A.A. Solodkov $^{127}$, O.V. Solovyanov ${ }^{127}$, V. Solovyev ${ }^{120}$, N. Soni ${ }^{85}$, V. Sopko ${ }^{126}$, B. Sopko ${ }^{126}$, M. Sosebee ${ }^{7}$, R. Soualah ${ }^{163 a, 163 c}$, A. Soukharev ${ }^{106}$, S. Spagnolo ${ }^{71 a, 71 b}$, F. Spanò ${ }^{75}$, R. Spighi ${ }^{19 a}$, G. Spigo ${ }^{29}$, R. Spiwoks ${ }^{29}$, M. Spousta ${ }^{125, \text { ah }}$, T. Spreitzer ${ }^{157}$, B. Spurlock ${ }^{7}$, R.D. St. Denis ${ }^{52}$, J. Stahlman ${ }^{119}$, R. Stamen ${ }^{57 a}$, E. Stanecka ${ }^{38}$, R.W. Stanek ${ }^{5}$, C. Stanescu ${ }^{133 a}$, M. StanescuBellu $^{41}$, S. Stapnes ${ }^{116}$, E.A. Starchenko ${ }^{127}$, J. Stark ${ }^{54}$, P. Staroba ${ }^{124}$, P. Starovoitov ${ }^{41}$, R. Staszewski ${ }^{38}$, A. Staude ${ }^{97}$, P. Stavina ${ }^{143 a, *}$, G. Steele ${ }^{52}$, P. Steinbach ${ }^{43}$, P. Steinberg ${ }^{24}$, I. Stekl ${ }^{126}$, B. Stelzer ${ }^{141}$, H.J. Stelzer ${ }^{87}$, O. Stelzer-Chilton ${ }^{158 a}$, H. Stenzel ${ }^{51}$, S. Stern ${ }^{98}$, G.A. Stewart ${ }^{29}$, J.A. Stillings ${ }^{20}$, M.C. Stockton ${ }^{84}$, K. Stoerig ${ }^{47}$, G. Stoicea ${ }^{25 a}$, S. Stonjek ${ }^{98}$, P. Strachota ${ }^{125}$, A.R. Stradling ${ }^{7}$, A. Straessner ${ }^{43}$, J. Strandberg ${ }^{146}$, S. Strandberg ${ }^{145 a, 145 b}$, A. Strandlie ${ }^{116}$, M. Strang ${ }^{108}$, 
E. Strauss ${ }^{142}$, M. Strauss ${ }^{110}$, P. Strizenec ${ }^{143 b}$, R. Ströhmer ${ }^{173}$, D.M. Strom ${ }^{113}$, J.A. Strong ${ }^{75, *}$, R. Stroynowski ${ }^{39}$, J. Strube ${ }^{128}$, B. Stugu ${ }^{13}$, I. Stumer ${ }^{24, *}$, J. Stupak ${ }^{147}$, P. Sturm ${ }^{174}$, N.A. Styles ${ }^{41}$, D.A. Soh ${ }^{150, w}$, D. Su ${ }^{142}$, HS. Subramania ${ }^{2}$, A. Succurro $^{11}$, Y. Sugaya ${ }^{115}$, C. Suhr ${ }^{105}$, M. Suk ${ }^{125}$, V.V. Sulin ${ }^{93}$, S. Sultansoy ${ }^{3 d}$, T. Sumida ${ }^{66}$, X. Sun ${ }^{54}$, J.E. Sundermann ${ }^{47}$, K. Suruliz ${ }^{138}$, G. Susinno ${ }^{36 a, 36 b}$, M.R. Sutton ${ }^{148}$, Y. Suzuki ${ }^{64}$, Y. Suzuki ${ }^{65}$, M. Svatos ${ }^{124}$, S. Swedish ${ }^{167}$, I. Sykora ${ }^{143 a}$, T. Sykora ${ }^{125}$, J. Sánchez ${ }^{166}$, D. Ta ${ }^{104}$, K. Tackmann ${ }^{41}$, A. Taffard ${ }^{162}$, R. Tafirout ${ }^{158 a}$, N. Taiblum ${ }^{152}$, Y. Takahashi ${ }^{100}$, H. Takai ${ }^{24}$, R. Takashima ${ }^{67}$, H. Takeda ${ }^{65}$, T. Takeshita ${ }^{139}$, Y. Takubo ${ }^{64}$, M. Talby ${ }^{82}$, A. Talyshev ${ }^{106, f}$, M.C. Tamsett ${ }^{24}$, J. Tanaka ${ }^{154}$, R. Tanaka ${ }^{114}$, S. Tanaka ${ }^{130}$, S. Tanaka ${ }^{64}$, A.J. Tanasijczuk ${ }^{141}$, K. Tani ${ }^{65}$, N. Tannoury ${ }^{82}$, S. Tapprogge ${ }^{80}$, D. $\operatorname{Tardif}^{157}$, S. Tarem ${ }^{151}$, F. Tarrade ${ }^{28}$, G.F. Tartarelli ${ }^{88 a}$, P. Tas ${ }^{125}$, M. Tasevsky ${ }^{124}$, E. Tassi ${ }^{36 a, 36 b}$, M. Tatarkhanov ${ }^{14}$, Y. Tayalati ${ }^{134 \mathrm{~d}}$, C. Taylor $^{76}$, F.E. Taylor ${ }^{91}$, G.N. Taylor ${ }^{85}$, W. Taylor ${ }^{158 b}$, M. Teinturier ${ }^{114}$, M. Teixeira Dias Castanheira ${ }^{74}$, P. Teixeira-Dias ${ }^{75}$, K.K. Temming ${ }^{47}$, H. Ten Kate ${ }^{29}$, P.K. Teng ${ }^{150}$, S. Terada ${ }^{64}$, K. Terashi ${ }^{154}$, J. Terron ${ }^{79}$, M. Testa ${ }^{46}$, R.J. Teuscher ${ }^{157, \mathrm{k}}$, J. Therhaag ${ }^{20}$, T. Theveneaux-Pelzer ${ }^{77}$, S. Thoma ${ }^{47}$, J.P. Thomas ${ }^{17}$, E.N. Thompson ${ }^{34}$, P.D. Thomp$\operatorname{son}^{17}$, P.D. Thompson ${ }^{157}$, A.S. Thompson ${ }^{52}$, L.A. Thomsen ${ }^{35}$, E. Thomson ${ }^{119}$, M. Thomson ${ }^{27}$, W.M. Thong ${ }^{85}$, R.P. Thun ${ }^{86}$,

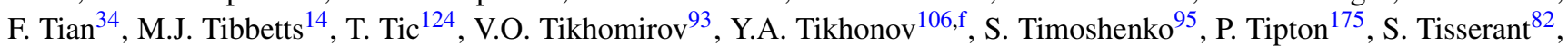

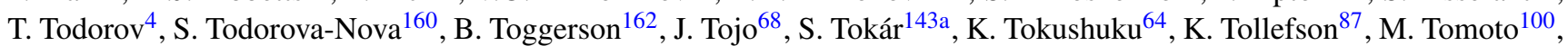
L. Tompkins ${ }^{30}$, K. Toms ${ }^{102}$, A. Tonoyan ${ }^{13}$, C. Topfel ${ }^{16}$, N.D. Topilinn ${ }^{63}$, I. Torchiani ${ }^{29}$, E. Torrence ${ }^{113}$, H. Torres $^{77}$, E. Torró Pastor $^{166}$, J. Toth ${ }^{82, \text { ad }}$, F. Touchard ${ }^{82}$, D.R. Tovey ${ }^{138}$, T. Trefzger ${ }^{173}$, L. Tremblet ${ }^{29}$, A. Tricoli ${ }^{29}$, I.M. Trigger ${ }^{158 a}$, S. Trincaz-

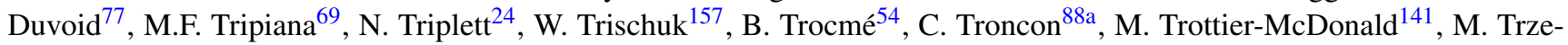
binski $^{38}$, A. Trzupek ${ }^{38}$, C. Tsarouchas ${ }^{29}$, J.C-L. Tseng ${ }^{117}$, M. Tsiakiris ${ }^{104}$, P.V. Tsiareshka ${ }^{89}$, D. Tsionou ${ }^{4, \text { ai }}$, G. Tsipolitis $^{9}$, S. Tsiskaridze ${ }^{11}$, V. Tsiskaridze ${ }^{47}$, E.G. Tskhadadze ${ }^{50 a}$, I.I. Tsukerman ${ }^{94}$, V. Tsulaia ${ }^{14}$, J.-W. Tsung ${ }^{20}$, S. Tsuno ${ }^{64}$,

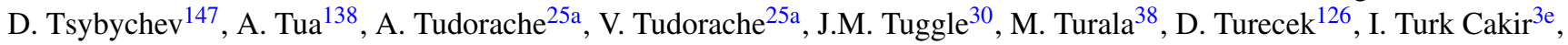

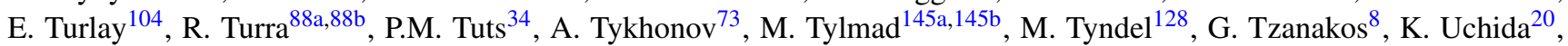
I. Ueda ${ }^{154}$, R. Ueno ${ }^{28}$, M. Ugland ${ }^{13}$, M. Uhlenbrock ${ }^{20}$, M. Uhrmacher ${ }^{53}$, F. Ukegawa ${ }^{159}$, G. Unal ${ }^{29}$, A. Undrus ${ }^{24}$, G. Unel ${ }^{162}$, Y. Unno ${ }^{64}$, D. Urbaniec ${ }^{34}$, G. Usai ${ }^{7}$, M. Uslenghi ${ }^{118 a, 118 b}$, L. Vacavant ${ }^{82}$, V. Vacek ${ }^{126}$, B. Vachon ${ }^{84}$, S. Vahsen $^{14}$, J. Valenta $^{124}$, S. Valentinetti ${ }^{19 a, 19 b}$, A. Valero ${ }^{166}$, S. Valkar ${ }^{125}$, E. Valladolid Gallego ${ }^{166}$, S. Vallecorsa ${ }^{151}$, J.A. Valls Ferrer ${ }^{166}$, R. Van Berg ${ }^{119}$, P.C. Van Der Deijl ${ }^{104}$, R. van der Geer ${ }^{104}$, H. van der Graaf ${ }^{104}$, R. Van Der Leeuw ${ }^{104}$, E. van der Poel ${ }^{104}$, D. van der $\operatorname{Ster}^{29}$, N. van Eldik ${ }^{29}$, P. van Gemmeren ${ }^{5}$, I. van Vulpen ${ }^{104}$, M. Vanadia ${ }^{98}$, W. Vandelli ${ }^{29}$, A. Vaniachine ${ }^{5}$, P. Vankov ${ }^{41}$, F. Vannucci ${ }^{77}$, R. Vari ${ }^{131 a}$, T. Varol ${ }^{83}$, D. Varouchas ${ }^{14}$, A. Vartapetian ${ }^{7}$, K.E. Varvell ${ }^{149}$, V.I. Vassilakopoulos ${ }^{55}$, F. Vazeille ${ }^{33}$, T. Vazquez Schroeder $^{53}$, G. Vegni ${ }^{88 a, 88 b}$, J.J. Veillet ${ }^{114}$, F. Veloso ${ }^{123 a}$, R. Veness ${ }^{29}$, S. Veneziano ${ }^{131 a}$, A. Ven-

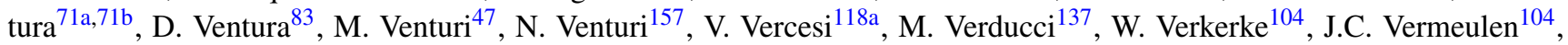
A. Vest ${ }^{43}$, M.C. Vetterli ${ }^{141, d}$, I. Vichou ${ }^{164}$, T. Vickey ${ }^{144 b, a j}$, O.E. Vickey Boeriu ${ }^{144 b}$, G.H.A. Viehhauser ${ }^{117}$, S. Viel ${ }^{167}$, M. Villa ${ }^{19 a, 19 b}$, M. Villaplana Perez ${ }^{166}$, E. Vilucchi ${ }^{46}$, M.G. Vincter ${ }^{28}$, E. Vinek ${ }^{29}$, V.B. Vinogradov ${ }^{63}$, M. Virchaux ${ }^{135, *}$, J. Virzi ${ }^{14}$, O. Vitells ${ }^{171}$, M. Viti ${ }^{41}$, I. Vivarelli ${ }^{47}$, F. Vives Vaque ${ }^{2}$, S. Vlachos ${ }^{9}$, D. Vladoiu ${ }^{97}$, M. Vlasak ${ }^{126}$, A. Vogel ${ }^{20}$, P. Vokac ${ }^{126}$, G. Volpi ${ }^{46}$, M. Volpi ${ }^{85}$, G. Volpini ${ }^{88 a}$, H. von der Schmitt $^{98}$, H. von Radziewski ${ }^{47}$, E. von Toerne ${ }^{20}$, V. Vorobel $^{125}$, V. Vorwerk ${ }^{11}$, M. Vos ${ }^{166}$, R. Voss ${ }^{29}$, T.T. Voss ${ }^{174}$, J.H. Vossebeld ${ }^{72}$, N. Vranjes ${ }^{135}$, M. Vranjes Milosavljevic ${ }^{104}$, V. Vrba ${ }^{124}$, M. Vreeswijk ${ }^{104}$, T. Vu Anh ${ }^{47}$, R. Vuillermet ${ }^{29}$, I. Vukotic ${ }^{30}$, W. Wagner ${ }^{174}$, P. Wagner ${ }^{119}$, H. Wahlen ${ }^{174}$, S. Wahrmund ${ }^{43}$, J. Wakabayashi ${ }^{100}$, S. Walch ${ }^{86}$, J. Walder ${ }^{70}$, R. Walker ${ }^{97}$, W. Walkowiak ${ }^{140}$, R. Wall ${ }^{175}$, P. Waller ${ }^{72}$, B. Walsh ${ }^{175}$, C. Wang ${ }^{44}$, H. Wang ${ }^{172}$, H. Wang ${ }^{32 b, a k}$, J. Wang ${ }^{150}$, J. Wang ${ }^{54}$, R. Wang ${ }^{102}$, S.M. Wang ${ }^{150}$, T. Wang ${ }^{20}$, A. Warburton ${ }^{84}$, C.P. Ward ${ }^{27}$, M. Warsinsky ${ }^{47}$, A. Washbrook ${ }^{45}$, C. Wasicki ${ }^{41}$, I. Watanabe ${ }^{65}$, P.M. Watkins ${ }^{17}$, A.T. Wat$\operatorname{son}^{17}$, I.J. Watson ${ }^{149}$, M.F. Watson ${ }^{17}$, G. Watts ${ }^{137}$, S. Watts ${ }^{81}$, A.T. Waugh ${ }^{149}$, B.M. Waugh ${ }^{76}$, M. Weber ${ }^{128}$, M.S. Weber $^{16}$, P. Weber ${ }^{53}$, A.R. Weidberg ${ }^{17}$, P. Weigell ${ }^{98}$, J. Weingarten ${ }^{53}$, C. Weiser ${ }^{47}$, H. Wellenstein ${ }^{22}$, P.S. Wells ${ }^{29}$, T. Wenaus $^{24}$, D. Wendland ${ }^{15}$, Z. Weng ${ }^{150, w}$, T. Wengler ${ }^{29}$, S. Wenig ${ }^{29}$, N. Wermes ${ }^{20}$, M. Werner ${ }^{47}$, P. Werner ${ }^{29}$, M. Werth $^{162}$, M. Wessels ${ }^{57 a}$, J. Wetter ${ }^{160}$, C. Weydert ${ }^{54}$, K. Whalen ${ }^{28}$, S.J. Wheeler-Ellis ${ }^{162}$, A. White ${ }^{7}$, M.J. White ${ }^{85}$, S. White ${ }^{121 a, 121 b}$, S.R. Whitehead ${ }^{117}$, D. Whiteson ${ }^{162}$, D. Whittington ${ }^{59}$, F. Wicek ${ }^{114}$, D. Wicke ${ }^{174}$, F.J. Wickens ${ }^{128}$, W. Wiedenmann ${ }^{172}$, M. Wielers ${ }^{128}$, P. Wienemann ${ }^{20}$, C. Wiglesworth ${ }^{74}$, L.A.M. Wiik-Fuchs ${ }^{47}$, P.A. Wijeratne ${ }^{76}$, A. Wildauer ${ }^{166}$, M.A. Wildt ${ }^{41, \mathrm{~s}}$, I. Wilhelm ${ }^{125}$, H.G. Wilkens ${ }^{29}$, J.Z. Will ${ }^{97}$, E. Williams ${ }^{34}$, H.H. Williams ${ }^{119}$, W. Willis ${ }^{34}$, S. Willocq ${ }^{83}$, J.A. Wilson ${ }^{17}$, M.G. Wilson ${ }^{142}$, A. Wilson ${ }^{86}$, I. Wingerter-Seez ${ }^{4}$, S. Winkelmann ${ }^{47}$, F. Winklmeier ${ }^{29}$, M. Wittgen ${ }^{142}$, S.J. Wollstadt ${ }^{80}$, M.W. Wolter ${ }^{38}$, H. Wolters ${ }^{123 a, h}$, W.C. Wong ${ }^{40}$, G. Wooden ${ }^{86}$, B.K. Wosiek ${ }^{38}$, J. Wotschack ${ }^{29}$, M.J. Woudstra ${ }^{81}$, K.W. Woz-

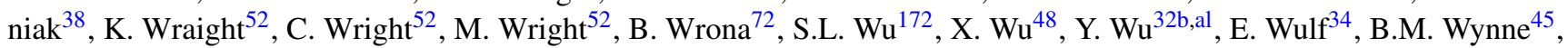
S. Xella ${ }^{35}$, M. Xiao ${ }^{135}$, S. Xie ${ }^{47}$, C. Xu ${ }^{32 b, z}$, D. Xu ${ }^{138}$, B. Yabsley ${ }^{149}$, S. Yacoob ${ }^{144 b}$, M. Yamada ${ }^{64}$, H. Yamaguchi $^{154}$, A. Yamamoto ${ }^{64}$, K. Yamamoto ${ }^{62}$, S. Yamamoto ${ }^{154}$, T. Yamamura ${ }^{154}$, T. Yamanaka ${ }^{154}$, J. Yamaoka ${ }^{44}$, T. Yamazaki ${ }^{154}$,

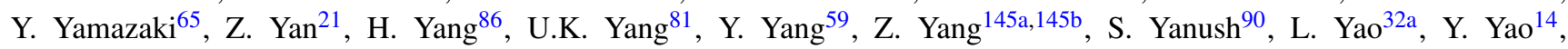
Y. Yasu ${ }^{64}$, G.V. Ybeles Smit ${ }^{129}$, J. Ye ${ }^{39}$, S. Ye ${ }^{24}$, M. Yilmaz ${ }^{3 c}$, R. Yoosoofmiya ${ }^{122}$, K. Yorita ${ }^{170}$, R. Yoshida ${ }^{5}$, C. Young ${ }^{142}$, C.J. Young ${ }^{117}$, S. Youssef ${ }^{21}$, D. $\mathrm{Yu}^{24}$, J. Yu ${ }^{7}$, J. Yu ${ }^{111}$, L. Yuan ${ }^{65}$, A. Yurkewicz ${ }^{105}$, M. Byszewski ${ }^{29}$, B. Zabinski ${ }^{38}$, 
R. Zaidan ${ }^{61}$, A.M. Zaitsev ${ }^{127}$, Z. Zajacova ${ }^{29}$, L. Zanello ${ }^{131 a, 131 b}$, A. Zaytsev ${ }^{106}$, C. Zeitnitz ${ }^{174}$, M. Zeman ${ }^{124}$, A. Zemla ${ }^{38}$, C. Zendler ${ }^{20}$, O. Zenin ${ }^{127}$, T. Ženiš ${ }^{143 a}$, Z. Zinonos ${ }^{121 a, 121 b}$, S. Zenz ${ }^{14}$, D. Zerwas ${ }^{114}$, G. Zevi della Porta ${ }^{56}$, Z. Zhan ${ }^{32 d}$, D. Zhang ${ }^{32 b, a k}$, H. Zhang ${ }^{87}$, J. Zhang ${ }^{5}$, X. Zhang ${ }^{32 \mathrm{~d}}$, Z. Zhang ${ }^{114}$, L. Zhao ${ }^{107}$, T. Zhao ${ }^{137}$, Z. Zhao ${ }^{32 b}$, A. Zhemchugov ${ }^{63}$, J. Zhong ${ }^{117}$, B. Zhou ${ }^{86}$, N. Zhou ${ }^{162}$, Y. Zhou ${ }^{150}$, C.G. Zhu ${ }^{32 d}$, H. Zhu ${ }^{41}$, J. Zhu ${ }^{86}$, Y. Zhu ${ }^{32 b}$, X. Zhuang ${ }^{97}$, V. Zhuravlov ${ }^{98}$, D. Zieminska ${ }^{59}$, N.I. Zimin ${ }^{63}$, R. Zimmermann ${ }^{20}$, S. Zimmermann ${ }^{20}$, S. Zimmermann ${ }^{47}$, M. Ziolkowski ${ }^{140}$, R. Zitoun ${ }^{4}$, L. Živković ${ }^{34}$, V.V. Zmouchko ${ }^{127, *}$, G. Zobernig ${ }^{172}$, A. Zoccoli ${ }^{19 a, 19 b}$, M. zur Nedden ${ }^{15}$, V. Zutshi ${ }^{105}$, L. Zwalinski ${ }^{29}$

${ }^{1}$ Physics Department, SUNY Albany, Albany NY, United States of America

${ }^{2}$ Department of Physics, University of Alberta, Edmonton AB, Canada

${ }^{3(a)}$ Department of Physics, Ankara University, Ankara; ${ }^{(b)}$ Department of Physics, Dumlupinar University, Kutahya;

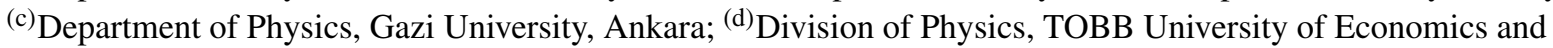
Technology, Ankara; ${ }^{(e)}$ Turkish Atomic Energy Authority, Ankara, Turkey

${ }^{4}$ LAPP, CNRS/IN2P3 and Université de Savoie, Annecy-le-Vieux, France

${ }^{5}$ High Energy Physics Division, Argonne National Laboratory, Argonne IL, United States of America

${ }^{6}$ Department of Physics, University of Arizona, Tucson AZ, United States of America

${ }^{7}$ Department of Physics, The University of Texas at Arlington, Arlington TX, United States of America

${ }^{8}$ Physics Department, University of Athens, Athens, Greece

${ }^{9}$ Physics Department, National Technical University of Athens, Zografou, Greece

${ }^{10}$ Institute of Physics, Azerbaijan Academy of Sciences, Baku, Azerbaijan

${ }^{11}$ Institut de Física d'Altes Energies and Departament de Física de la Universitat Autònoma de Barcelona and ICREA, Barcelona, Spain

12(a) Institute of Physics, University of Belgrade, Belgrade; (b) Vinca Institute of Nuclear Sciences, University of Belgrade,

Belgrade, Serbia

${ }^{13}$ Department for Physics and Technology, University of Bergen, Bergen, Norway

${ }^{14}$ Physics Division, Lawrence Berkeley National Laboratory and University of California, Berkeley CA, United States of America

${ }^{15}$ Department of Physics, Humboldt University, Berlin, Germany

${ }^{16}$ Albert Einstein Center for Fundamental Physics and Laboratory for High Energy Physics, University of Bern, Bern, Switzerland

${ }^{17}$ School of Physics and Astronomy, University of Birmingham, Birmingham, United Kingdom

18(a) Department of Physics, Bogazici University, Istanbul; ${ }^{(\mathrm{b})}$ Division of Physics, Dogus University, Istanbul;

${ }^{(c)}$ Department of Physics Engineering, Gaziantep University, Gaziantep; ${ }^{(d)}$ Department of Physics, Istanbul Technical University, Istanbul, Turkey

19(a) INFN Sezione di Bologna; ${ }^{(b)}$ Dipartimento di Fisica, Università di Bologna, Bologna, Italy

${ }^{20}$ Physikalisches Institut, University of Bonn, Bonn, Germany

${ }^{21}$ Department of Physics, Boston University, Boston MA, United States of America

${ }^{22}$ Department of Physics, Brandeis University, Waltham MA, United States of America

23(a) Universidade Federal do Rio De Janeiro COPPE/EE/IF, Rio de Janeiro; ${ }^{(b)}$ Federal University of Juiz de Fora (UFJF), Juiz de Fora; ${ }^{\left({ }^{c}\right)}$ Federal University of Sao Joao del Rei (UFSJ), Sao Joao del Rei; ${ }^{(d)}$ Instituto de Fisica, Universidade de Sao Paulo, Sao Paulo, Brazil

${ }^{24}$ Physics Department, Brookhaven National Laboratory, Upton NY, United States of America

25(a) National Institute of Physics and Nuclear Engineering, Bucharest; ${ }^{(b)}$ University Politehnica Bucharest, Bucharest;

${ }^{(c)}$ West University in Timisoara, Timisoara, Romania

${ }^{26}$ Departamento de Física, Universidad de Buenos Aires, Buenos Aires, Argentina

${ }^{27}$ Cavendish Laboratory, University of Cambridge, Cambridge, United Kingdom

${ }^{28}$ Department of Physics, Carleton University, Ottawa ON, Canada

${ }^{29} \mathrm{CERN}$, Geneva, Switzerland

${ }^{30}$ Enrico Fermi Institute, University of Chicago, Chicago IL, United States of America

31(a) Departamento de Física, Pontificia Universidad Católica de Chile, Santiago; ${ }^{(b)}$ Departamento de Física, Universidad Técnica Federico Santa María, Valparaíso, Chile

32(a) Institute of High Energy Physics, Chinese Academy of Sciences, Beijing; ${ }^{(b)}$ Department of Modern Physics, University of Science and Technology of China, Anhui; ${ }^{(c)}$ Department of Physics, Nanjing University, Jiangsu; ${ }^{(d)}$ School of Physics, Shandong University, Shandong, China 
${ }^{33}$ Laboratoire de Physique Corpusculaire, Clermont Université and Université Blaise Pascal and CNRS/IN2P3, Clermont-Ferrand, France

${ }^{34}$ Nevis Laboratory, Columbia University, Irvington NY, United States of America

${ }^{35}$ Niels Bohr Institute, University of Copenhagen, Kobenhavn, Denmark

36(a) INFN Gruppo Collegato di Cosenza; ${ }^{\left({ }^{b}\right)}$ Dipartimento di Fisica, Università della Calabria, Arcavata di Rende, Italy

${ }^{37}$ AGH University of Science and Technology, Faculty of Physics and Applied Computer Science, Krakow, Poland

${ }^{38}$ The Henryk Niewodniczanski Institute of Nuclear Physics, Polish Academy of Sciences, Krakow, Poland

${ }^{39}$ Physics Department, Southern Methodist University, Dallas TX, United States of America

${ }^{40}$ Physics Department, University of Texas at Dallas, Richardson TX, United States of America

${ }^{41}$ DESY, Hamburg and Zeuthen, Germany

${ }^{42}$ Institut für Experimentelle Physik IV, Technische Universität Dortmund, Dortmund, Germany

${ }^{43}$ Institut für Kern- und Teilchenphysik, Technical University Dresden, Dresden, Germany

${ }^{44}$ Department of Physics, Duke University, Durham NC, United States of America

${ }^{45}$ SUPA - School of Physics and Astronomy, University of Edinburgh, Edinburgh, United Kingdom

${ }^{46}$ INFN Laboratori Nazionali di Frascati, Frascati, Italy

${ }^{47}$ Fakultät für Mathematik und Physik, Albert-Ludwigs-Universität, Freiburg, Germany

${ }^{48}$ Section de Physique, Université de Genève, Geneva, Switzerland

49(a) INFN Sezione di Genova; ${ }^{(b)}$ Dipartimento di Fisica, Università di Genova, Genova, Italy

${ }^{50(a)}$ E. Andronikashvili Institute of Physics, Tbilisi State University, Tbilisi; ${ }^{(b)}$ High Energy Physics Institute, Tbilisi State University, Tbilisi, Georgia

${ }^{51}$ II Physikalisches Institut, Justus-Liebig-Universität Giessen, Giessen, Germany

${ }^{52}$ SUPA - School of Physics and Astronomy, University of Glasgow, Glasgow, United Kingdom

${ }^{53}$ II Physikalisches Institut, Georg-August-Universität, Göttingen, Germany

${ }^{54}$ Laboratoire de Physique Subatomique et de Cosmologie, Université Joseph Fourier and CNRS/IN2P3 and Institut National Polytechnique de Grenoble, Grenoble, France

${ }^{55}$ Department of Physics, Hampton University, Hampton VA, United States of America

${ }^{56}$ Laboratory for Particle Physics and Cosmology, Harvard University, Cambridge MA, United States of America

57(a) Kirchhoff-Institut für Physik, Ruprecht-Karls-Universität Heidelberg, Heidelberg; (b) Physikalisches Institut,

Ruprecht-Karls-Universität Heidelberg, Heidelberg; ${ }^{(c)}$ ZITI Institut für technische Informatik,

Ruprecht-Karls-Universität Heidelberg, Mannheim, Germany

${ }^{58}$ Faculty of Applied Information Science, Hiroshima Institute of Technology, Hiroshima, Japan

${ }^{59}$ Department of Physics, Indiana University, Bloomington IN, United States of America

${ }^{60}$ Institut für Astro- und Teilchenphysik, Leopold-Franzens-Universität, Innsbruck, Austria

${ }^{61}$ University of Iowa, Iowa City IA, United States of America

${ }^{62}$ Department of Physics and Astronomy, Iowa State University, Ames IA, United States of America

${ }^{63}$ Joint Institute for Nuclear Research, JINR Dubna, Dubna, Russia

${ }^{64}$ KEK, High Energy Accelerator Research Organization, Tsukuba, Japan

${ }^{65}$ Graduate School of Science, Kobe University, Kobe, Japan

${ }^{66}$ Faculty of Science, Kyoto University, Kyoto, Japan

${ }^{67}$ Kyoto University of Education, Kyoto, Japan

${ }^{68}$ Department of Physics, Kyushu University, Fukuoka, Japan

${ }^{69}$ Instituto de Física La Plata, Universidad Nacional de La Plata and CONICET, La Plata, Argentina

${ }^{70}$ Physics Department, Lancaster University, Lancaster, United Kingdom

71(a) INFN Sezione di Lecce; ${ }^{\text {(b) }}$ Dipartimento di Matematica e Fisica, Università del Salento, Lecce, Italy

${ }^{72}$ Oliver Lodge Laboratory, University of Liverpool, Liverpool, United Kingdom

${ }^{73}$ Department of Physics, Jožef Stefan Institute and University of Ljubljana, Ljubljana, Slovenia

${ }^{74}$ School of Physics and Astronomy, Queen Mary University of London, London, United Kingdom

${ }^{75}$ Department of Physics, Royal Holloway University of London, Surrey, United Kingdom

${ }^{76}$ Department of Physics and Astronomy, University College London, London, United Kingdom

${ }^{77}$ Laboratoire de Physique Nucléaire et de Hautes Energies, UPMC and Université Paris-Diderot and CNRS/IN2P3, Paris, France

${ }^{78}$ Fysiska institutionen, Lunds universitet, Lund, Sweden

${ }^{79}$ Departamento de Fisica Teorica C-15, Universidad Autonoma de Madrid, Madrid, Spain 
${ }^{80}$ Institut für Physik, Universität Mainz, Mainz, Germany

${ }^{81}$ School of Physics and Astronomy, University of Manchester, Manchester, United Kingdom

${ }^{82} \mathrm{CPPM}$, Aix-Marseille Université and CNRS/IN2P3, Marseille, France

${ }^{83}$ Department of Physics, University of Massachusetts, Amherst MA, United States of America

${ }^{84}$ Department of Physics, McGill University, Montreal QC, Canada

${ }^{85}$ School of Physics, University of Melbourne, Victoria, Australia

${ }^{86}$ Department of Physics, The University of Michigan, Ann Arbor MI, United States of America

${ }^{87}$ Department of Physics and Astronomy, Michigan State University, East Lansing MI, United States of America

${ }^{88(a)}$ INFN Sezione di Milano; ${ }^{\text {(b) }}$ Dipartimento di Fisica, Università di Milano, Milano, Italy

${ }^{89}$ B.I. Stepanov Institute of Physics, National Academy of Sciences of Belarus, Minsk, Republic of Belarus

${ }^{90}$ National Scientific and Educational Centre for Particle and High Energy Physics, Minsk, Republic of Belarus

${ }^{91}$ Department of Physics, Massachusetts Institute of Technology, Cambridge MA, United States of America

${ }^{92}$ Group of Particle Physics, University of Montreal, Montreal QC, Canada

${ }^{93}$ P.N. Lebedev Institute of Physics, Academy of Sciences, Moscow, Russia

${ }^{94}$ Institute for Theoretical and Experimental Physics (ITEP), Moscow, Russia

${ }^{95}$ Moscow Engineering and Physics Institute (MEPhI), Moscow, Russia

${ }^{96}$ Skobeltsyn Institute of Nuclear Physics, Lomonosov Moscow State University, Moscow, Russia

${ }^{97}$ Fakultät für Physik, Ludwig-Maximilians-Universität München, München, Germany

${ }^{98}$ Max-Planck-Institut für Physik (Werner-Heisenberg-Institut), München, Germany

${ }^{99}$ Nagasaki Institute of Applied Science, Nagasaki, Japan

${ }^{100}$ Graduate School of Science and Kobayashi-Maskawa Institute, Nagoya University, Nagoya, Japan

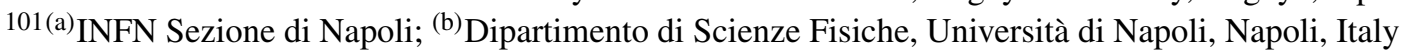

${ }^{102}$ Department of Physics and Astronomy, University of New Mexico, Albuquerque NM, United States of America

${ }^{103}$ Institute for Mathematics, Astrophysics and Particle Physics, Radboud University Nijmegen/Nikhef, Nijmegen, Netherlands

${ }^{104}$ Nikhef National Institute for Subatomic Physics and University of Amsterdam, Amsterdam, Netherlands

${ }^{105}$ Department of Physics, Northern Illinois University, DeKalb IL, United States of America

${ }^{106}$ Budker Institute of Nuclear Physics, SB RAS, Novosibirsk, Russia

${ }^{107}$ Department of Physics, New York University, New York NY, United States of America

${ }^{108}$ Ohio State University, Columbus OH, United States of America

${ }^{109}$ Faculty of Science, Okayama University, Okayama, Japan

${ }^{110}$ Homer L. Dodge Department of Physics and Astronomy, University of Oklahoma, Norman OK, United States of America

${ }^{111}$ Department of Physics, Oklahoma State University, Stillwater OK, United States of America

${ }^{112}$ Palacký University, RCPTM, Olomouc, Czech Republic

${ }^{113}$ Center for High Energy Physics, University of Oregon, Eugene OR, United States of America

${ }^{114}$ LAL, Université Paris-Sud and CNRS/IN2P3, Orsay, France

${ }^{115}$ Graduate School of Science, Osaka University, Osaka, Japan

${ }^{116}$ Department of Physics, University of Oslo, Oslo, Norway

${ }^{117}$ Department of Physics, Oxford University, Oxford, United Kingdom

${ }^{118(a)}$ INFN Sezione di Pavia; ${ }^{(b)}$ Dipartimento di Fisica, Università di Pavia, Pavia, Italy

${ }^{119}$ Department of Physics, University of Pennsylvania, Philadelphia PA, United States of America

${ }^{120}$ Petersburg Nuclear Physics Institute, Gatchina, Russia

121 (a) INFN Sezione di Pisa; ${ }^{(b)}$ Dipartimento di Fisica E. Fermi, Università di Pisa, Pisa, Italy

${ }^{122}$ Department of Physics and Astronomy, University of Pittsburgh, Pittsburgh PA, United States of America

${ }^{123(a)}$ Laboratorio de Instrumentacao e Fisica Experimental de Particulas - LIP, Lisboa, Portugal; ${ }^{(b)}$ Departamento de Fisica

Teorica y del Cosmos and CAFPE, Universidad de Granada, Granada, Spain

${ }^{124}$ Institute of Physics, Academy of Sciences of the Czech Republic, Praha, Czech Republic

${ }^{125}$ Faculty of Mathematics and Physics, Charles University in Prague, Praha, Czech Republic

${ }^{126}$ Czech Technical University in Prague, Praha, Czech Republic

${ }^{127}$ State Research Center Institute for High Energy Physics, Protvino, Russia

${ }^{128}$ Particle Physics Department, Rutherford Appleton Laboratory, Didcot, United Kingdom

${ }^{129}$ Physics Department, University of Regina, Regina SK, Canada 
${ }^{130}$ Ritsumeikan University, Kusatsu, Shiga, Japan

131(a) INFN Sezione di Roma I; ${ }^{(b)}$ Dipartimento di Fisica, Università La Sapienza, Roma, Italy

132(a) INFN Sezione di Roma Tor Vergata; ${ }^{(b)}$ Dipartimento di Fisica, Università di Roma Tor Vergata, Roma, Italy

133(a) INFN Sezione di Roma Tre; ${ }^{(b)}$ Dipartimento di Fisica, Università Roma Tre, Roma, Italy

${ }^{134(a)}$ Faculté des Sciences Ain Chock, Réseau Universitaire de Physique des Hautes Energies - Université Hassan II,

Casablanca; ${ }^{(b)}$ Centre National de l'Energie des Sciences Techniques Nucleaires, Rabat; ${ }^{(c)}$ Faculté des Sciences

Semlalia, Université Cadi Ayyad, LPHEA, Marrakech; ${ }^{(d)}$ Faculté des Sciences, Université Mohamed Premier and

LPTPM, Oujda; ${ }^{(e)}$ Faculté des sciences, Université Mohammed V-Agdal, Rabat, Morocco

${ }^{135}$ DSM/IRFU (Institut de Recherches sur les Lois Fondamentales de l'Univers), CEA Saclay (Commissariat a l'Energie

Atomique), Gif-sur-Yvette, France

${ }^{136}$ Santa Cruz Institute for Particle Physics, University of California Santa Cruz, Santa Cruz CA, United States of America

${ }^{137}$ Department of Physics, University of Washington, Seattle WA, United States of America

${ }^{138}$ Department of Physics and Astronomy, University of Sheffield, Sheffield, United Kingdom

${ }^{139}$ Department of Physics, Shinshu University, Nagano, Japan

${ }^{140}$ Fachbereich Physik, Universität Siegen, Siegen, Germany

${ }^{141}$ Department of Physics, Simon Fraser University, Burnaby BC, Canada

${ }^{142}$ SLAC National Accelerator Laboratory, Stanford CA, United States of America

${ }^{143(a)}$ Faculty of Mathematics, Physics \& Informatics, Comenius University, Bratislava; ${ }^{(b)}$ Department of Subnuclear

Physics, Institute of Experimental Physics of the Slovak Academy of Sciences, Kosice, Slovak Republic

${ }^{144(a)}$ Department of Physics, University of Johannesburg, Johannesburg; ${ }^{\left({ }^{b}\right)}$ School of Physics, University of the

Witwatersrand, Johannesburg, South Africa

145(a) Department of Physics, Stockholm University; ${ }^{(b)}$ The Oskar Klein Centre, Stockholm, Sweden

${ }^{146}$ Physics Department, Royal Institute of Technology, Stockholm, Sweden

${ }^{147}$ Departments of Physics \& Astronomy and Chemistry, Stony Brook University, Stony Brook NY, United States of

America

${ }^{148}$ Department of Physics and Astronomy, University of Sussex, Brighton, United Kingdom

${ }^{149}$ School of Physics, University of Sydney, Sydney, Australia

${ }^{150}$ Institute of Physics, Academia Sinica, Taipei, Taiwan

${ }^{151}$ Department of Physics, Technion: Israel Institute of Technology, Haifa, Israel

${ }^{152}$ Raymond and Beverly Sackler School of Physics and Astronomy, Tel Aviv University, Tel Aviv, Israel

${ }^{153}$ Department of Physics, Aristotle University of Thessaloniki, Thessaloniki, Greece

${ }^{154}$ International Center for Elementary Particle Physics and Department of Physics, The University of Tokyo, Tokyo, Japan

${ }^{155}$ Graduate School of Science and Technology, Tokyo Metropolitan University, Tokyo, Japan

${ }^{156}$ Department of Physics, Tokyo Institute of Technology, Tokyo, Japan

${ }^{157}$ Department of Physics, University of Toronto, Toronto ON, Canada

158(a) TRIUMF, Vancouver BC; ${ }^{(b)}$ Department of Physics and Astronomy, York University, Toronto ON, Canada

${ }^{159}$ Faculty of Pure and Applied Sciences, University of Tsukuba, Tsukuba, Japan

${ }^{160}$ Department of Physics and Astronomy, Tufts University, Medford MA, United States of America

${ }^{161}$ Centro de Investigaciones, Universidad Antonio Narino, Bogota, Colombia

${ }^{162}$ Department of Physics and Astronomy, University of California Irvine, Irvine CA, United States of America

163(a) INFN Gruppo Collegato di Udine, Udine; ${ }^{(b)}$ ICTP, Trieste; ${ }^{(c)}$ Dipartimento di Chimica, Fisica e Ambiente, Università

di Udine, Udine, Italy

${ }^{164}$ Department of Physics, University of Illinois, Urbana IL, United States of America

${ }^{165}$ Department of Physics and Astronomy, University of Uppsala, Uppsala, Sweden

${ }^{166}$ Instituto de Física Corpuscular (IFIC) and Departamento de Física Atómica, Molecular y Nuclear and Departamento de Ingeniería Electrónica and Instituto de Microelectrónica de Barcelona (IMB-CNM), University of Valencia and CSIC, Valencia, Spain

${ }^{167}$ Department of Physics, University of British Columbia, Vancouver BC, Canada

${ }^{168}$ Department of Physics and Astronomy, University of Victoria, Victoria BC, Canada

${ }^{169}$ Department of Physics, University of Warwick, Coventry, United Kingdom

${ }^{170}$ Waseda University, Tokyo, Japan

${ }^{171}$ Department of Particle Physics, The Weizmann Institute of Science, Rehovot, Israel

${ }^{172}$ Department of Physics, University of Wisconsin, Madison WI, United States of America 
${ }^{173}$ Fakultät für Physik und Astronomie, Julius-Maximilians-Universität, Würzburg, Germany

${ }^{174}$ Fachbereich C Physik, Bergische Universität Wuppertal, Wuppertal, Germany

${ }^{175}$ Department of Physics, Yale University, New Haven CT, United States of America

${ }^{176}$ Yerevan Physics Institute, Yerevan, Armenia

${ }^{177}$ Centre de Calcul de l'Institut National de Physique Nucléaire et de Physique des Particules (IN2P3), Villeurbanne,

France

${ }^{a}$ Also at Laboratorio de Instrumentacao e Fisica Experimental de Particulas - LIP, Lisboa, Portugal

bAlso at Faculdade de Ciencias and CFNUL, Universidade de Lisboa, Lisboa, Portugal

${ }^{\mathrm{c}}$ Also at Particle Physics Department, Rutherford Appleton Laboratory, Didcot, United Kingdom

${ }^{\mathrm{d}}$ Also at TRIUMF, Vancouver BC, Canada

e Also at Department of Physics, California State University, Fresno CA, United States of America

${ }_{\mathrm{f}}^{\mathrm{f}}$ Also at Novosibirsk State University, Novosibirsk, Russia

${ }^{\mathrm{g}}$ Also at Fermilab, Batavia IL, United States of America

${ }^{\mathrm{h}}$ Also at Department of Physics, University of Coimbra, Coimbra, Portugal

${ }^{\mathrm{i} A l s o}$ at Department of Physics, UASLP, San Luis Potosi, Mexico

$\mathrm{j}$ Also at Università di Napoli Parthenope, Napoli, Italy

${ }^{k}$ Also at Institute of Particle Physics (IPP), Canada

${ }^{1}$ Also at Department of Physics, Middle East Technical University, Ankara, Turkey

${ }^{m}$ Also at Louisiana Tech University, Ruston LA, United States of America

${ }^{\mathrm{n}}$ Also at Dep Fisica and CEFITEC of Faculdade de Ciencias e Tecnologia, Universidade Nova de Lisboa, Caparica, Portugal

${ }^{\circ}$ Also at Department of Physics and Astronomy, University College London, London, United Kingdom

pAlso at Group of Particle Physics, University of Montreal, Montreal QC, Canada

${ }^{\mathrm{q}}$ Also at Department of Physics, University of Cape Town, Cape Town, South Africa

${ }^{\mathrm{r}}$ Also at Institute of Physics, Azerbaijan Academy of Sciences, Baku, Azerbaijan

${ }^{\mathrm{s}}$ Also at Institut für Experimentalphysik, Universität Hamburg, Hamburg, Germany

${ }^{t}$ Also at Manhattan College, New York NY, United States of America

"Also at School of Physics, Shandong University, Shandong, China

${ }^{v}$ Also at CPPM, Aix-Marseille Université and CNRS/IN2P3, Marseille, France

${ }^{\mathrm{w}}$ Also at School of Physics and Engineering, Sun Yat-sen University, Guanzhou, China

${ }^{x}$ Also at Academia Sinica Grid Computing, Institute of Physics, Academia Sinica, Taipei, Taiwan

${ }^{y}$ Also at Dipartimento di Fisica, Università La Sapienza, Roma, Italy

${ }^{\mathrm{z}} \mathrm{Also}$ at DSM/IRFU (Institut de Recherches sur les Lois Fondamentales de l'Univers), CEA Saclay (Commissariat a l'Energie Atomique), Gif-sur-Yvette, France

${ }^{\text {aa }}$ Also at Section de Physique, Université de Genève, Geneva, Switzerland

${ }^{a b}$ Also at Departamento de Fisica, Universidade de Minho, Braga, Portugal

${ }^{a c}$ Also at Department of Physics and Astronomy, University of South Carolina, Columbia SC, United States of America

${ }^{a d}$ Also at Institute for Particle and Nuclear Physics, Wigner Research Centre for Physics, Budapest, Hungary

${ }^{\text {ae }}$ Also at California Institute of Technology, Pasadena CA, United States of America

af Also at Institute of Physics, Jagiellonian University, Krakow, Poland

${ }^{a g}$ Also at LAL, Université Paris-Sud and CNRS/IN2P3, Orsay, France

${ }^{\text {ah }}$ Also at Nevis Laboratory, Columbia University, Irvington NY, United States of America

ai Also at Department of Physics and Astronomy, University of Sheffield, Sheffield, United Kingdom

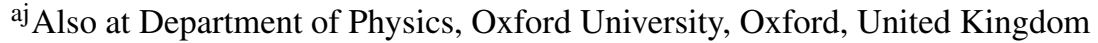

${ }^{a k}$ Also at Institute of Physics, Academia Sinica, Taipei, Taiwan

${ }^{\text {al }}$ Also at Department of Physics, The University of Michigan, Ann Arbor MI, United States of America

*Deceased 